\title{
Metamagnetic texture in a polar antiferromagnet
}

\author{
D. A. Sokolov*, ${ }^{1}$ N. Kikugawa, ${ }^{2}$ T. Helm, ${ }^{1}$ H. Borrmann, ${ }^{1}$ U. Burkhardt, ${ }^{1}$ R. Cubitt, ${ }^{3}$ J. S. \\ White, ${ }^{4}$ E. Ressouche, ${ }^{5}$ M. Bleuel, ${ }^{6,7}$ K. Kummer, ${ }^{8}$ A. P. Mackenzie, ${ }^{1,9}$ and U. K. Rößler ${ }^{10}$ \\ ${ }^{I}$ Max-Planck-Institut für Chemische Physik fester Stoffe, D-01187 Dresden, Germany \\ ${ }^{2}$ National Institute for Materials Science, Tsukuba 305-0003, Japan \\ ${ }^{3}$ Institut Laue-Langevin, 6 Rue Jules Horowitz, F-38042 Grenoble, France \\ ${ }^{4}$ Laboratory for Neutron Scattering and Imaging (LNS), \\ Paul Scherrer Institute (PSI), CH-5232 Villigen, Switzerland \\ ${ }^{5}$ Univ. Grenoble Alpes, CEA, INAC-MEM, 38000 Grenoble, France \\ ${ }^{6}$ NIST Center for Neutron Research National Institute of Standards and Technology Gaithersburg, MD 20988-8562, USA \\ ${ }^{7}$ Department of Materials Science and Engineering University of Maryland, College Park, MD 20742-2115, USA \\ ${ }^{8}$ ESRF, 71 avenue des Martyrs, 38000 Grenoble, France \\ ${ }^{9}$ Scottish Universities Physics Alliance (SUPA), School of Physics and Astronomy, \\ University of St Andrews, St Andrews KY16 9SS, United Kingdom \\ ${ }^{10}$ IFW Dresden, PO Box 270116, D-01171 Dresden, Germany
}

\begin{abstract}
The notion of a simple ordered state implies homogeneity. If the order is established by a broken symmetry, the elementary Landau theory of phase transitions shows that only one symmetry mode describes this state. Precisely at points of phase coexistence domain states formed of large regions of different phases can be stabilized by long range interactions. In uniaxial antiferromagnets the so-called metamagnetism is an example of such a behavior, when antiferromagnetic and field-induced spin-polarized paramagnetic/ferromagnetic states co-exist at a jump-like transition in the magnetic phase diagram. Here, combining experiment with theoretical analysis, we show that a different type of mixed state between antiferromagnetism and ferromagnetism can be created in certain non-centrosymmetric materials. In the small-angle neutron scattering experiments we observe a field-driven spin-state in the layered antiferromagnet $\mathrm{Ca}_{3} \mathrm{Ru}_{2} \mathrm{O}_{7}$, which is modulated on a scale between 8 and $20 \mathrm{~nm}$ and has both antiferromagnetic and ferromagnetic parts. We call this state a metamagnetic texture and explain its appearance by the chiral twisting effects of the asymmetric Dzyaloshinskii-Moriya (DM) exchange. The observation can be understood as an extraordinary coexistence, in one thermodynamic state, of spin-orders belonging to different symmetries. Experimentally, the complex nature of this metamagnetic state is demonstrated by measurements of anomalies in electronic transport which reflect the spin-polarization in the metamagnetic texture, determination of the magnetic orbital moments, which supports the existence of strong spin-orbit effects, a pre-requisite for the mechanism of twisted magnetic states in this material. Our findings provide an example of a rich and largely unexplored class of textured states. Such textures mediate between different ordering modes near phase co-existence, and engender extremely rich phase diagrams.
\end{abstract}

\section{INTRODUCTION}

The term metamagnetism, possibly coined by Kramers as a joke, was used to describe the bizarre properties of certain magnetic materials that have been investigated for more than 100 years. They appeared to be paramagnetic or antiferromagnetic in the ground state, but ferromagnetic in applied fields[1-3]. Metamagnetism now labels a sudden rise or crossover of the magnetization under applied field and is observed in various classes of materials. Once Néel's notion of antiferromagnetism had been accepted, one type of metamagnetic behavior could easily be explained as the jump-like transition between a collinear antiferromagnetic up-down state and a spin-polarized up-up state when a field overcomes the exchange between sublattice spins constrained to collinear configurations by a strong easy-axis magnetic anisotropy [3, 4]. In the generic magnetic phase diagrams of such materials, a first-order phase transition occurs between the two spinorders. Long-range classical dipolar interactions or magnetostrictive interactions can stabilize domain states in which the two spin-orders co-exist. These classical domain structures at phase-coexistence points are well understood [5].

Ordered states with rotatable order parameters may also display intrinsically inhomogeneous phases. Here twisting short-range forces cause a continuous modification of the order-parameter direction. In non-centrosymmetric magnetically ordered materials, spin-orbit effects on the magnetic exchange interactions cause chiral spiral ordering[6]. Phenomenological theory is able to predict and describe such modulated states in a wide range of condensed matter systems, such as incommensurable states in certain crystals undergoing lattice instabilities [7,8], or chiral liquid crystals [9-11]. In such modulated textures the direction of a multicomponent order parameter spatially rotates from one orientation to another. Chiral helimagnetic order is a paragon of such textures in which spin-orbit coupling twists an elementary spin-ordered pattern over long periods [6, 12, 13]. For this type of directional order in systems with a twisting short-range force, static multidimensional solitons are theoretically predicted[14], which now are called chiral skyrmions in the case of chiral ferromagnets or Néel antiferromagnets [15]. The condensation of such particle-like states can yield rich phase diagrams [16, 17], which have become a major topic in condensed matter magnetism over the last decade $[18,19]$. Although a chiral helimagnet macroscopically behaves as an antiferromagnet, the primary magnetic order is a simple ferromagnetic spin order, twisted into a helix over long distances $[12,13]$. 
Here we show how a spiral magnetic order emerges in a material with antiferromagnetic order parameter. The spiral propagates in a direction perpendicular to the wavevector of antiferromagnetic order. Materials displaying such complex textures may also host new types of antiferromagnetic skyrmions, which are a subject of intense theoretical and experimental research[20]. We present the first experimental realization of a magnetic texture composed of an antiferromagnetic ground state and a ferromagnetic spin-polarized state. We identified the layered orthorhombic antiferromagnetic oxide $\mathrm{Ca}_{3} \mathrm{Ru}_{2} \mathrm{O}_{7}$, as suitable for a focused search for a metamagnetic texture. This material crystallizes in the noncentrosymmetric polar structure described by space-group $B b 2_{1} m$, which belongs to polar point-group $C_{2 v}$. The crystal structure consists of $\mathrm{RuO}_{2}$ bilayers with corner sharing $\mathrm{RuO}_{6}$ octahedra, which are rotated around the crystallographic $c$ axis and tilted with respect to the $a b$-plane[21]. The basic antiferromagnetic order-parameter in $\mathrm{Ca}_{3} \mathrm{Ru}_{2} \mathrm{O}_{7}$ was identified in detailed neutron diffraction studies[22]. This magnetic-order parameter is described by a simple collinear ordering-mode, which does not allow for a canting of moments into a weak ferromagnetic state. Thus, $\mathrm{Ca}_{3} \mathrm{Ru}_{2} \mathrm{O}_{7}$ meets the elementary symmetry conditions for modulated magnetism. A further requirement is relevant spin-orbit couplings, that affect the primary magnetic order. We have used X-ray magnetic circular dichroism (XMCD) spectroscopy on the Ru ions to measure its orbital magnetic moment. We find relatively large moments with a ratio of orbital to spin moment of about 0.15 , see SFIG. 1 in Supplementary materials in agreement with earlier [23] and our own theoretical investigations. This indicates that possibly strong antisymmetric $\mathrm{DM}$ exchange interactions do affect the magnetic order in $\mathrm{Ca}_{3} \mathrm{Ru}_{2} \mathrm{O}_{7}$. The material orders antiferromagnetically below the Néel temperature, $T_{N}=56 \mathrm{~K}$ with the ordered moments along the $a$-axis and the magnetic propagation vector along the [001] direction. Within the bilayer the Ru moments are coupled ferromagnetically, whereas the coupling between the adjacent bilayers is antiferromagnetic [21, 22]. This state is normally referred to as AFM-a. On cooling below $48 \mathrm{~K}$, the ordered moments within the bilayer spontaneously re-orient to point along the $b$-axis, and this state is known as AFM-b. The coupling between the adjacent bilayers remains antiferromagnetic. The moment re-orientation is accompanied by the first order structural transition at 48 $\mathrm{K}[24]$.

The isothermal magnetization at low temperatures displays a single metamagnetic transition and reaches $\sim 1.95 \mu_{B}$ per $\mathrm{Ru}$ ion, a slightly reduced value compared to $2 \mu_{B}$ for the full moment expected for $\mathrm{Ru}^{4+}$, FIG 1 a. At $\mathrm{T} \geq 43 \mathrm{~K}$ the magnetization shows two metamagnetic transitions, which become increasingly separated in field as the temperature increased to $48 \mathrm{~K}$, resulting in the "funnel"-type structure in the magnetic susceptibility, $\mathrm{dm} / \mathrm{dH}$ plotted as a function of temperature and magnetic field, FIG $1 \mathrm{~b}$. The higher field transition at $\mathrm{H}>5.5 \mathrm{~T}$ in the magnetization is a transition into a canted antiferromagnetic structure, CAFM, in which the magnetic moments on $\mathrm{Ru}$ ions are partially polarized along the direction of the applied field[22]. We have also detected a small hysteresis at the low field transition, a typical signature of metamagnetism. Fur- ther below we show that the fields and temperatures at which the hysteresis was observed mark the onset of metamagnetic texture observed in small-angle neutron scattering (SANS) experiments.

The metamagnetic transitions between the antiferromagnetic ground states with mutually orthogonal directions of the staggered magnetization have strong signatures in the specific heat measurements, FIG 1c. A sharp, lambda-like transition in the specific heat in zero field at $48 \mathrm{~K}$ is suppressed to lower temperature as the magnetic field increases up to $5.4 \mathrm{~T}$. At higher fields the transition becomes broader and is shifted to higher temperatures in agreement with the "funnel"-type structure, shown in FIG $1 \mathrm{~b}$.

The metamagnetic transitions also manifest in the electrical transport measurements, as shown in FIG 1d. The Hall resistivity $R_{x y}$ for current along the $a$-axis and with magnetic field along the $b$-axis displays two features that are marked by local maxima in the derivative $d R_{x y} / \mathrm{dH}$. These maxima split towards lower and higher fields upon increasing temperature, a behavior similar to $\mathrm{dm} / \mathrm{dH}$, see further discussion and SFIG.2,3 in Supplementary materials. Our analysis, following the general approach for the Anomalous Hall effect (AHE)[25], indicates that in addition to a strong AHE component there is an intrinsic additional contribution in the region between the two metamagnetic transitions, see Supplementary materials. This suggests the presence of a magnetic texture with either topological features or non-collinear complex modulations in this magnetic state that can contribute an extraordinary off-diagonal components of the resistivity tensor. A quantitative extraction of this extraordinary Hallresistivity may only become possible by taking into account field-induced changes in the band structure and the exact structure of the new magnetic order, and lies beyond the scope of this work.

The results of our bulk measurements refine the published phase diagram of $\mathrm{Ca}_{3} \mathrm{Ru}_{2} \mathrm{O}_{7}$ in the region between the lines separating AFM-b and CAFM states[26-28]. Until now most of the neutron scattering measurements on $\mathrm{Ca}_{3} \mathrm{Ru}_{2} \mathrm{O}_{7}$ were performed at commensurate wavevectors. In this Article we focus on the nature of the magnetic state near AFM-a to AFM$\mathrm{b}$ transition at magnetic fields between $2 \mathrm{~T}$ and $5 \mathrm{~T}$ and report the magnetic modulation in a previously unexplored region of the reciprocal space near the wavevector $Q=0$.

SANS experiments were performed to search directly for a bulk long-wavelength magnetic modulation in the "funnel"type region of the $\mu_{0} \mathrm{H}-\mathrm{T}$ phase diagram near $\mathrm{Q}=0$. Typical SANS patterns obtained at 4 different fields are shown in FIG 2. Each pattern consists of the images of the main two-dimensional low-Q detector and 4 additional high-Q detectors as detailed in Ref.[29]. The magnetic field was applied parallel to the $b$-axis, which points out of the plane of the detector. Data were collected after zero field cooling the sample to $2 \mathrm{~K}$, applying the field at $2 \mathrm{~K}$ and measuring the pattern at several increasing temperatures. For each field a non-magnetic background collected at $65 \mathrm{~K}$ was subtracted. The most striking feature found in our experiments is a pair of satellites at $\mathrm{Q}_{M M T}=( \pm \Delta, 0,0)$, which correspond to a magnetic modulation propagating along the a-axis with a repeat 
(a)
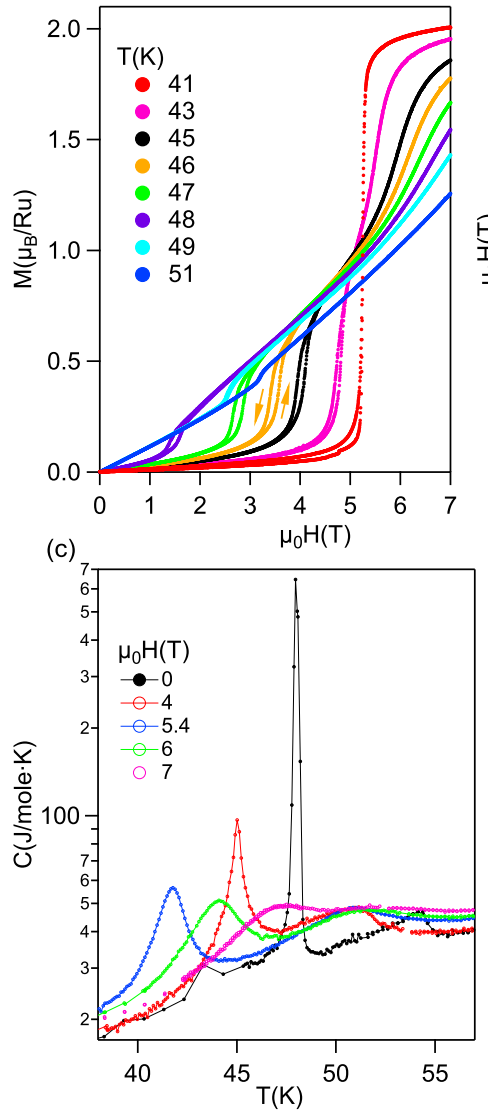

(b)
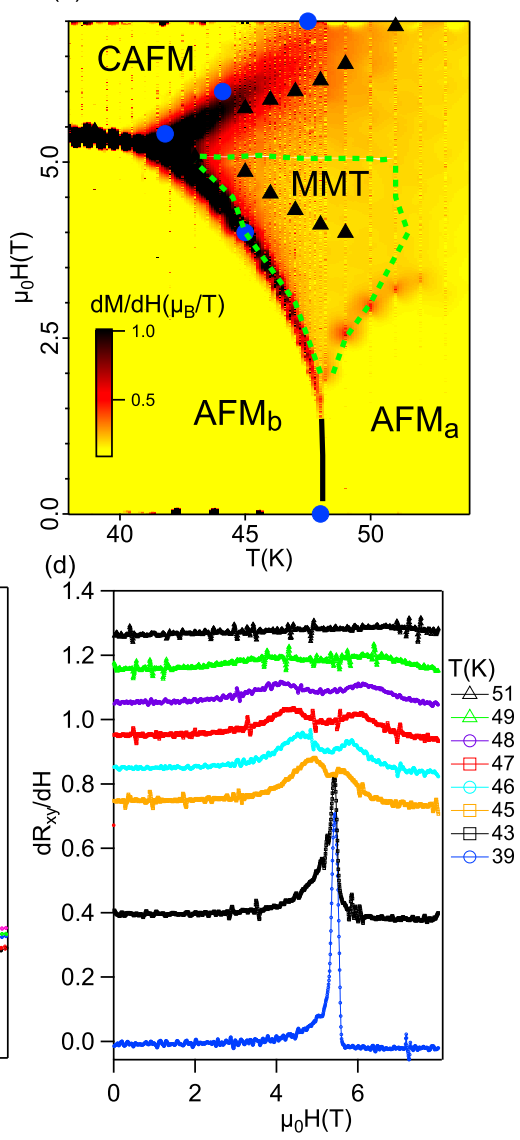

FIG. 1: Bulk properties of $\mathrm{Ca}_{3} \mathrm{Ru}_{2} \mathrm{O}_{7}$ measured with the magnetic field along the b-axis on the same single crystal: (a) Magnetic field dependent magnetization at various temperatures spanning the region of interest in the phase diagram. Up(down) arrow corresponds to increase (decrease) of the field. (b) Differential susceptibility $\mathrm{dm} / \mathrm{dH}$ obtained by differentiating magnetization in (a) with respect to the field. The color scale represents a magnitude of $\mathrm{dm} / \mathrm{dH}$. Dotted green line encircles the region in which the metamagnetic texture (MMT) was observed in SANS measurements, see text. Solid black line corresponds to transitions inferred from low field m(T) measurements. Black filled triangles in (b) correspond to the maximum in $\mathrm{dR}_{x y} / \mathrm{dH}$ in the Hall effect measurements. Blue filled dots in (b) are inferred from the field dependent maxima in the specific heat. AFM-a, AFM-b, and CAFM mark two antiferromagnetic and the canted antiferromagnetic regions of the phase diagram, see text. (c) Temperature dependence of the specific heat. The sharp maximum at zero field marks the moment re-orientation transition. (d) Derivative of the Hall resistivity with respect to the magnetic field.

distance of $2 \pi / \Delta$, FIG 2 . We observed the satellites at fields from $2 \mathrm{~T}$ up to $5 \mathrm{~T}$ in the temperature range, which shows a hysteretic behaviour of the magnetisation shown in FIG 1a. No satellites were observed at fields above $5 \mathrm{~T}$, suggesting that the "funnel"- type region of the phase diagram is not a uniform magnetically ordered state. The satellites develop from the strong intensity near $\mathrm{Q}=0$ at the temperature of AFM-a to AFM-b transition. The scattering is broad with respect to wavevector near the onset temperature, FIG 2a. The apparent diffuse nature of the scattering is most likely due to a quasilong-range ordering. The wavevector of satellites initially increases on heating, although in FIG 3 we show that the temperature dependence of the wavevector is not monotonic at all fields. This pattern was observed at all fields except for $2 \mathrm{~T}$, where satellites exist only in a very small temperature range in a proximity of the metamagnetic transition. We also observed a second harmonic of the primary satellites at $2 \mathrm{Q}_{M M T}$ at $2 \mathrm{~T}$,
2.5 $\mathrm{T}$ and $3 \mathrm{~T}$, which could correspond to higher order peaks or represent a double scattering.

The magnetic field and the wavevector dependence of SANS intensity is summarized in FIG 3. Increasing the magnetic field suppresses intensity of satellites at the corresponding temperatures, FIG 3a. The intensity of the satellites is the strongest at the lowest temperature at which we can resolve the satellites from the strong scattering near the direct beam. The wavevector of the modulation is strongly temperature dependent. For $\mu_{0} \mathrm{H} \geq 4 \mathrm{~T}$ it increases continuously with temperature from $\mathrm{Q}_{M M T}<0.045 \AA^{-1}$ at the onset temperature up to $\mathrm{Q}_{M M T}=0.08 \AA^{-1}$ at $\mathrm{T} \geq 50 \mathrm{~K}$. In contrast, for $\mu_{0} \mathrm{H} \leq 3$ $\mathrm{T} \mathrm{Q}_{M M T}$ displays a sharp maximum just above the onset temperature, FIG 3b. The repeat distance of the modulation, $\Delta=2 \pi / Q_{M M T}$ reaches $\sim 200 \AA$ at the lowest temperature of observation of the satellites at $5 \mathrm{~T}$. Near $50 \mathrm{~K}$, at $5 \mathrm{~T}$ the repeat distance decreases to $\sim 80 \AA$. The competing character of sev- 

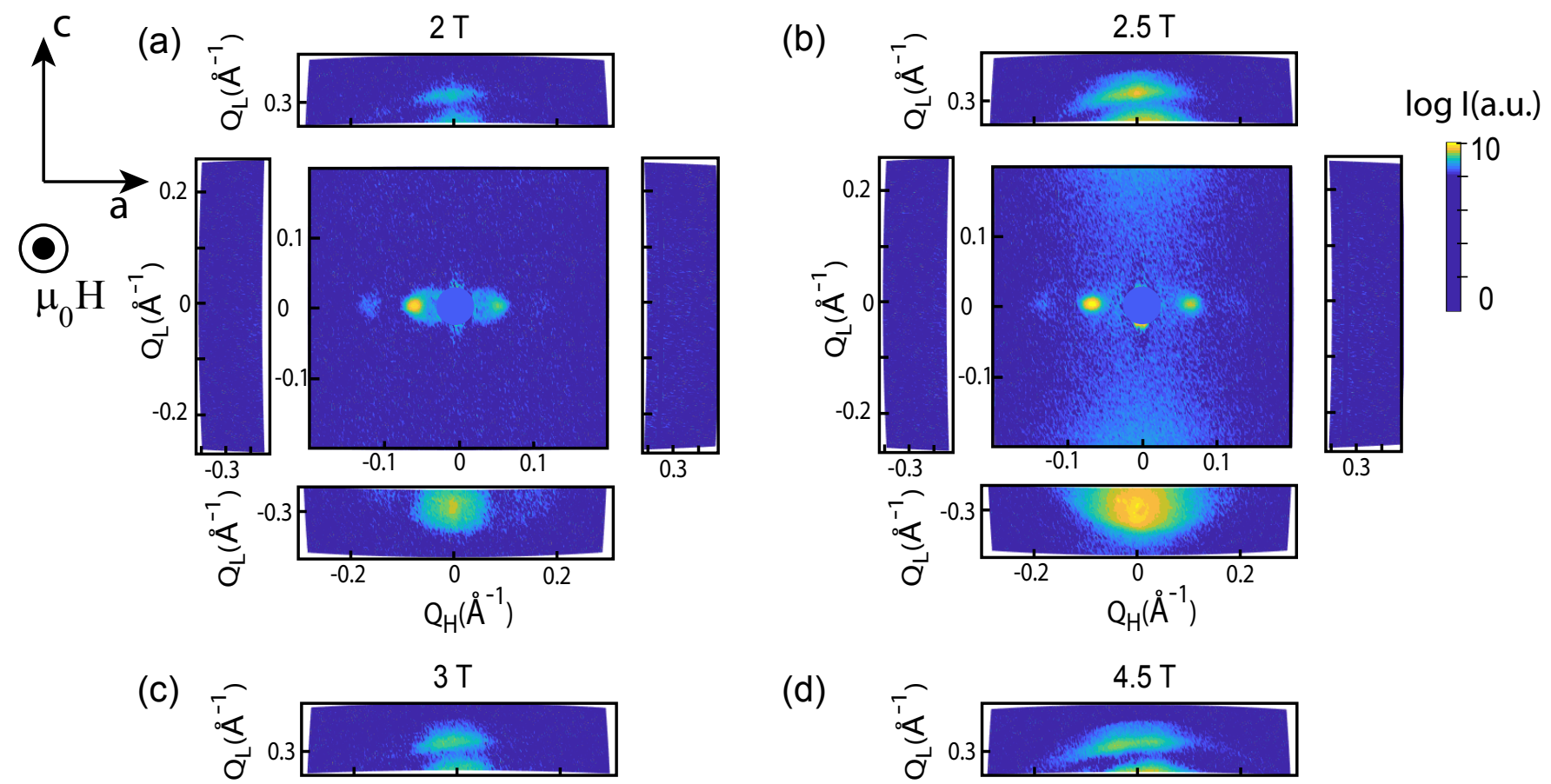

(d)
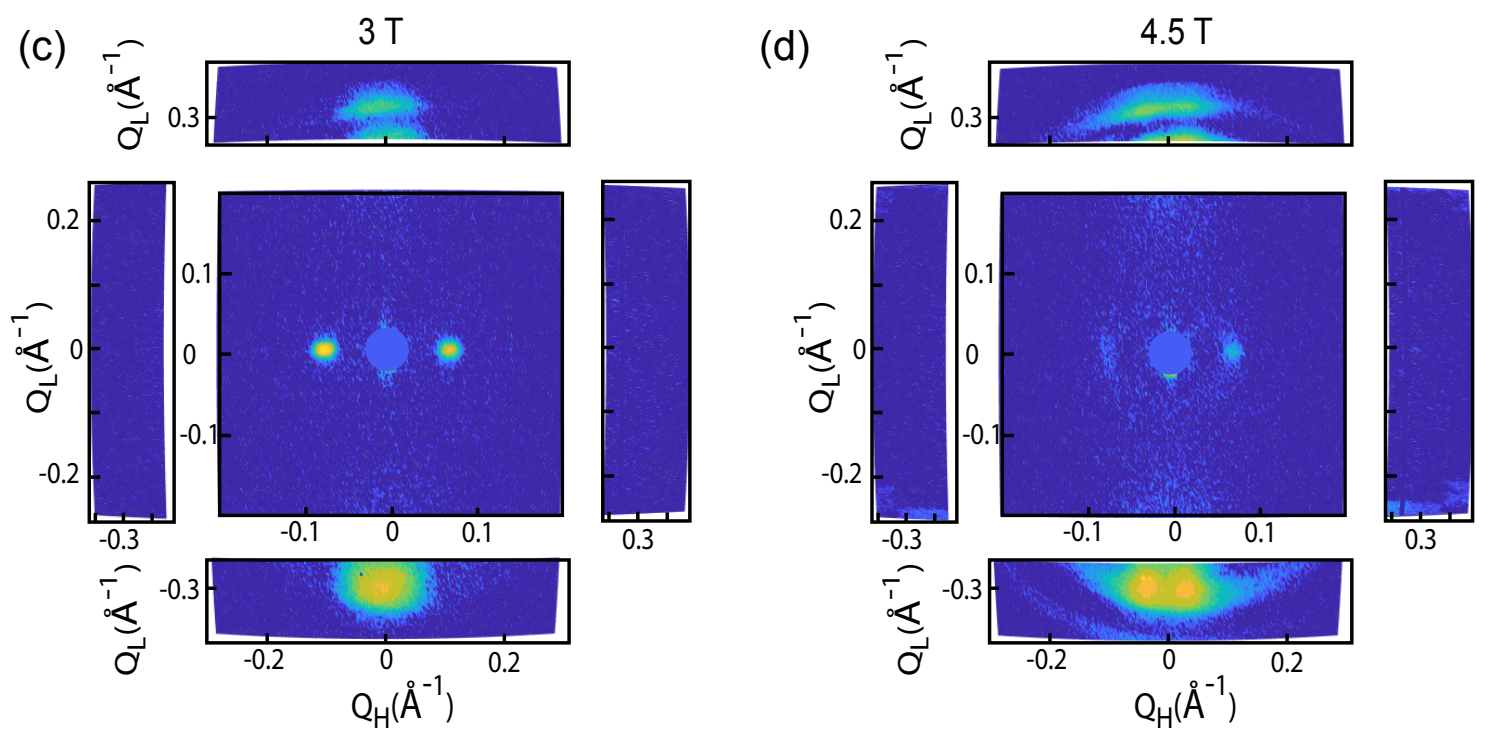

FIG. 2: Typical SANS patterns measured at $48 \mathrm{~K}$ in magnetic fields from $2 \mathrm{~T}$ (a) to $4.5 \mathrm{~T} \mathrm{(d)}$ applied parallel to the b-axis. 4 smaller panels are SANS detectors positioned at $1.2 \mathrm{~m}$ from the sample and thus able to detect diffraction from 001 magnetic reflection. The panel in the centre is the main SANS detector at $2 \mathrm{~m}$ from the sample. The metamagnetic texture (MMT) propagates along the $a$-axis with $\mathrm{Q}_{M M T}$ reaching $(0.08,0,0) \AA^{-1}$. Higher order reflections were observed at $2 \mathrm{~T}$ (a) and $2.5 \mathrm{~T}$ (b). A split along the $a$-axis reflection at $\mathrm{Q}_{A F}=(0,0,-0.32) \AA^{-1}$ was observed at $4.5 \mathrm{~T}(\mathrm{~d})$ and also at $5 \mathrm{~T}$, see Supplementary materials. No such splitting was observed below the onset of the metamagnetic texture, $\mathrm{T}_{M M T}$. Note the same logarithmic scale of the intensity for all fields. The non-magnetic background at $65 \mathrm{~K}$ measured at the corresponding field was subtracted from all the patterns. The filled blue circle at $\mathrm{Q}=0$ is a mask applied to cover the direct neutron beam. $\mathrm{Q}_{L}$ and $\mathrm{Q}_{H}$ are wavevectors along (00L) and (H00) directions.

eral coupling terms, which have different temperature dependencies, is most likely the origin of non-monotonic temperature dependence of the wavevector of the metamagnetic texture, see Supplemental materials for details. Using the rocking curve measurements we estimated the correlation length of the modulation along the $b$-axis, FIG $3 c$. For details of estimate, see SFIG.4 in supplementary materials. The correlation length $\xi_{b}$ is not resolution limited and reaches $2280 \AA$ at $2.5 \mathrm{~T}$, comparable to the correlation length of $5500 \AA$ in the A-phase of $\mathrm{MnSi}[18]$. Magnetic field suppresses correlations along $b$ axis at $\mu_{0} \mathrm{H}>2.5 \mathrm{~T}$. Neutron scattering is only sensitive to the component of the magnetization perpendicular to the total momentum transfer, so that the component of the ordered moment along the $a$-axis is not measured in our experiment. The observation of the satellites along the $a$-axis in the $a c$ plane indicates that the ordered moment of the modulation can have components parallel to the $c$ and $b$-axes. In AFM-b and AFM-a regions of the phase diagram, which border the region of the metamagnetic texture the ordered moment has no component along the $c$-axis. We also note that in none of the $\mathrm{Fe}$ 
and $\mathrm{Mn}$-doped $\mathrm{Ca}_{3} \mathrm{Ru}_{2} \mathrm{O}_{7}$ does the ordered moment acquire a component along the $c$-axis[30, 31]. It is therefore likely that the ordered moment of the modulation is along the $b$ axis, but the component along the $a$-axis cannot be ruled out. Our modulation is then either a helix or a cycloid if it has a component of the ordered moment along the propagation vector. Further experiments with polarized neutrons are required to identify the type of the modulation. The strong intensity near $\mathrm{Q}_{A F}=(0,0, \pm 0.32) \AA^{-1}$ or simply (001) corresponds to the bulk antiferromagnetism, which propagates along the $c$ axis in agreement with Ref.[22]. The scattering is very broad near $\mathrm{Q}_{A F}$ at the temperatures at which the satellites at $\mathrm{Q}_{M M T}$ are observed, but turns into a well-defined sharp reflection at the lowest temperatures, where no satellites are observed. The broad, ring-like shaped features near $\mathrm{Q}_{A F}$ possibly originate from a short-range or fluctuating antiferromagnetic order. We observed reflection at $\mathrm{Q}_{A F}=(00-1)$ only, no reflection was observed at $\mathrm{Q}_{A F}=(001)$ due to a small tilt of the crystal with respect to the vertical direction. We also note that the antiferromagnetic reflection acquires a modulation, which propagates along the $a$-axis, which becomes resolvable above $4 \mathrm{~T}$. The wavevector of the modulation, $\mathrm{Q}_{A F}^{m}=(\delta 0-1)$, where $\delta$ reaches $0.059 \AA^{-1}$ at $5 \mathrm{~T}$. This behaviour is reminiscent of a magnetic field-induced commensurate-to-incommensurate transition in the $\mathrm{DM}$ antiferromagnet $\mathrm{Ba}_{2} \mathrm{CuGe}_{2} \mathrm{O}_{7}$, in which the magnetic field was applied in the plane of the rotation of the spins[32]. A commensurate to incommensurate antiferromagnetic transition was also reported for $\mathrm{Fe}$ and $\mathrm{Mn}$-doped $\mathrm{Ca}_{3} \mathrm{Ru}_{2} \mathrm{O}_{7}$ in Ref.[30, 31]. A cycloidal modulation propagating along the $a$-axis was identified for both types of doping. These observations suggest that the antiferromagnetism in $\mathrm{Ca}_{3} \mathrm{Ru}_{2} \mathrm{O}_{7}$ can be easily destabilized by application of the magnetic field or doping and is prone to host magnetic solitons.

The onset of the magnetic texture with the ordered moment along $b$-axis requires the ordered moment in the $\mathrm{AFM}_{a}$ state to rotate from the $a$-axis to the $b$-axis locally, on the lengthscale of the magnetic texture. We propose that such defects in the magnetic structure break the long-range three-dimensional antiferromagnetism in $\mathrm{Ca}_{3} \mathrm{Ru}_{2} \mathrm{O}_{7}$. The intensity near the antiferromagnetic wavevectors $\mathrm{Q}_{A F}$ is maximised near the temperature at which the magnetic satellites at $\mathrm{Q}_{M M T}$ disappear. We note that in a previous neutron scattering work the intensity of the antiferromagnetic reflections measured between 3 and $4 \mathrm{~T}$ showed a reduced intensity on cooling [22]. We argue that the emergence of the magnetic texture is the origin of the reduced integrated intensity of the antiferromagnetic Bragg peaks at $\mathrm{Q}_{A F}$. It is unlikely therefore, that the magnetic texture and the bulk antiferromagnetism co-exist in a non-equilibrium state. Instead, the magnetic texture develops from the antiferromagnetism as an equilibrium state in the presence of the magnetic field, which enhances the effect of the DM interactions. Further neutron diffraction experiments are needed to describe the splitting of (001) magnetic reflection.

Summarizing the experiments, $\mathrm{Ca}_{3} \mathrm{Ru}_{2} \mathrm{O}_{7}$ displays a spirally-modulated magnetic order in broad temperature-field region, previously regarded as a crossover[22, 28]. The propagation vector of the spiral is aligned perpendicular to the mag- netic field and the staggered magnetization is likely parallel to it. The magnetic field applied along the polar $b$-axis destabilizes the antiferromagnetic ground state by flipping spins from the $a$-axis to $b$-axis locally on a scale between 8 and $20 \mathrm{~nm}$ depending on the magnetic field and temperature as illustrated schematically in FIG $4 \mathrm{~b}$. Then, a mixed state modulating between antiferromagnetic and ferromagnetic spinconfigurations with long periods, is observed. Our observation of metamagnetic textures in $\mathrm{Ca}_{3} \mathrm{Ru}_{2} \mathrm{O}_{7}$ invites a comparison with spiral spin states driven by a competition between direct exchange interactions such as in $\mathrm{Ca}_{3} \mathrm{Co}_{2} \mathrm{O}_{6}$ [33] and $\mathrm{MnSc}_{2} \mathrm{~S}_{4}$ [34] or rare-earth elements such as Tb, Dy, Ho, which demonstrate a helically modulated magnetic structure due to nesting of the Fermi surface or a Kohn anomaly[35]. Although the theoretical description of the helical modulation in $\mathrm{Ca}_{3} \mathrm{Co}_{2} \mathrm{O}_{6}$ is still lacking, it is considered to result from a competition of antiferromagnetic and ferromagnetic direct exchange interactions. A magnetic vortex state reported in $\mathrm{MnSc}_{2} \mathrm{~S}_{4}$ results most likely due to a competition between nearest and the next nearest neighbour exchange interactions[34]. Elemental rare-earths such as Tb, Dy, and Ho order via long-range exchange interactions carried by conduction electrons (RKKY) at a finite wavevector dictated by RKKY. As the temperature is lowered the effects of crystalline electric field and magnetic anisotropy lead to a reduction of the ordering wavevector and transition into ferromagnetic state. The theories explaining the magnetic structures of $\mathrm{Tb}$, Dy, Ho consider a high density of states near the Fermi level, which drives the nesting. The phenomenon of metamagnetic textures in $\mathrm{Ca}_{3} \mathrm{Ru}_{2} \mathrm{O}_{7}$ is distinctly different from both above mentioned examples as the textured state results from a coupling of ferromagnetic and antiferromagnetic order parameters via so-called Lifshitz invariants, see Supplementary materials for details. A rather generic character of such a term in the free-energy expansion suggests that more spin textured states driven by DM interaction are awaiting discovery.

Elementary considerations are sufficient to explain why this modulated state exists, and in fact is an expected behavior in a polar antiferromagnet such as $\mathrm{Ca}_{3} \mathrm{Ru}_{2} \mathrm{O}_{7}$ when, under a magnetic field, its magnetic states is transformed into a ferromagnetic configuration. Its non-centrosymmetric crystal structure and layered antiferromagnetic ordering (FIG 4a) enable specific couplings between the two co-existing order parameters, the antiferromagnetic staggered magnetization and the ferromagnetic spin polarization. Ultimately, these couplings derive from the DM interactions (DMI) in this material. The hierarchy between (i) the strong spin-exchange, stabilizing a certain antiferromagnetic spin-pattern as the ground state, (ii) the twisting effects of the DMIs on this ground-state, and (iii) the possibility to tune the system into the spin-polarized state by external magnetic fields, while temperature is used to tune the weaker magnetic anisotropies, makes this layered chiral magnet with a polar structure ideally suited for a modulation of the desired type.

In contrast to a "proper" Dzyaloshinskii texture where one directional ordering mode is twisted, this is a more complex texture generated near the co-existence points of two phases. Here, the magnetic order is spatially wavering between the 

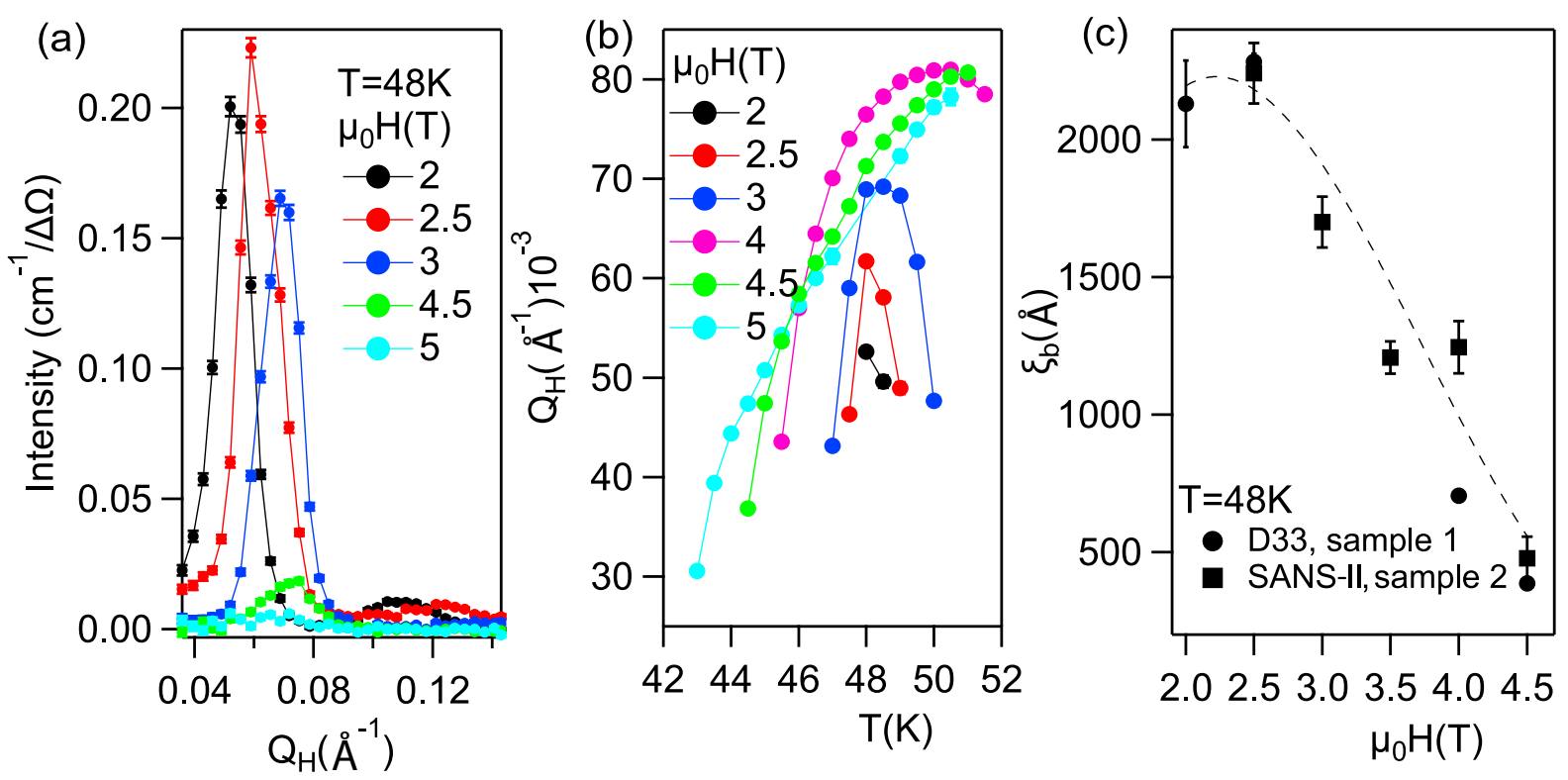

FIG. 3: Metamagnetic textures in $\mathrm{Ca}_{3} \mathrm{Ru}_{2} \mathrm{O}_{7}$ : (a) Magnetic field dependence of the wavevector-dependent azimuthally averaged SANS intensity on the detector plane capturing magnetic satellite at $\mathrm{Q}_{M M T}$ measured at $48 \mathrm{~K}$. (b) Temperature dependence of the wavevector of the modulation measured at fields from $2 \mathrm{~T}$ to $5 \mathrm{~T}$. (c) Magnetic field dependence of the correlation length along $b$-axis at $48 \mathrm{~K}$ measured in samples 1 and 2 on D33, ILL and on SANS-II, PSI, see Methods. Where not shown explicitly, the errorbars (one sigma standard deviation) are smaller than markers. Lines are a guide to the eye.

co-existing ferromagnetic and antiferrromagnetic configurations, as sketched in FIG 4b. Therefore, the term "metamagnetic texture" appears appropriate for this modulated phase in $\mathrm{Ca}_{3} \mathrm{Ru}_{2} \mathrm{O}_{7}$.

Qualitatively, the mechanism enabling such complex mixed states can be stated by using symmetries to construct the phenomenological continuums theory for these ordering modes, i.e. by constructing the Landau-Ginzburg free energy for the coexisting and coupled ordering modes as reported for $\mathrm{Ca}_{3} \mathrm{Ru}_{2} \mathrm{O}_{7}$ in the Supplementary materials. The specific mechanism then is described by free energy terms known as "Lifshitz-type invariants". Such terms are linear in spatial gradients of one mode and couple it to the other mode. These Lifshitz-type terms describe a frustrated coupling between different pure modes. The expectation that such terms cause modulations of thermodynamic mixed phases has been put forth theoretically for a long time [36-41]. However, these effects can become relevant only if a system can be tuned towards special multicritical regions of the phase diagram, where the two primary modes co-exist. This may be the reason why concrete examples for the effects of such terms have been scarce. Typically such effects have been discussed for frustrated (magnetic) systems of low symmetry where different order parameters already co-exist in the ground state[42]. Recently, the importance of such couplings has been raised in the context of "phase co-existence" in materials with multiple electronic instabilities such as the manganite or cuprate perovskites[41]. The phase diagrams of these materials may include multicritical points and also co-existence of ferro- and antiferromagnetic phases, but the role of Lifshitz-type couplings for mixed states is difficult to establish for the elec- tronic or structural order parameters. Hence, simple experimental systems displaying mixed textures composed of different ordering modes have remained elusive. The discovery of field-driven modulated magnetic state in $\mathrm{Ca}_{3} \mathrm{Ru}_{2} \mathrm{O}_{7}$ now provides an example of a modulated state with mixed symmetries.

The phenomenological theory describing its metamagnetic behavior is detailed in the section VI of the Supplemental materials. The Landau-Ginzburg free energy displays Lifshitztype invariants that are anisotropic in spin directions and favour modulated coupled states between FM and AFM spinstructure. The specific form of these terms reveals that they are caused by spin-orbit interactions and encode the twisting influence of the antisymmetric DM-exchange on the magnetic order.

The observations demonstrate that metamagnetic crystals with appropriate non-centrosymmetric structure are an ideal playground for creating such textures. As can be justified from the phenomenological Landau theory, the spin-twisting DMIs in these magnets preclude homogeneous phases, unless stabilized by additional strong anisotropies, and generically favour mixed AFM-FM states near the metamagnetic transition.

FIG 4c,d shows schematically possible magnetic phase diagrams that can be realized in antiferromagnets with noncentrosymmetric, in particular polar symmetry.

The tricritical region with first-order phase transitions in the case of magnets with strong easy-axis anisotropy is replaced by transitional regions covered by modulated mesophases. Similarly, for systems with weak or absent anisotropies, modulated states still can occur near the transition towards the spin-polarized paramagnetic phase at elevated temperatures, 

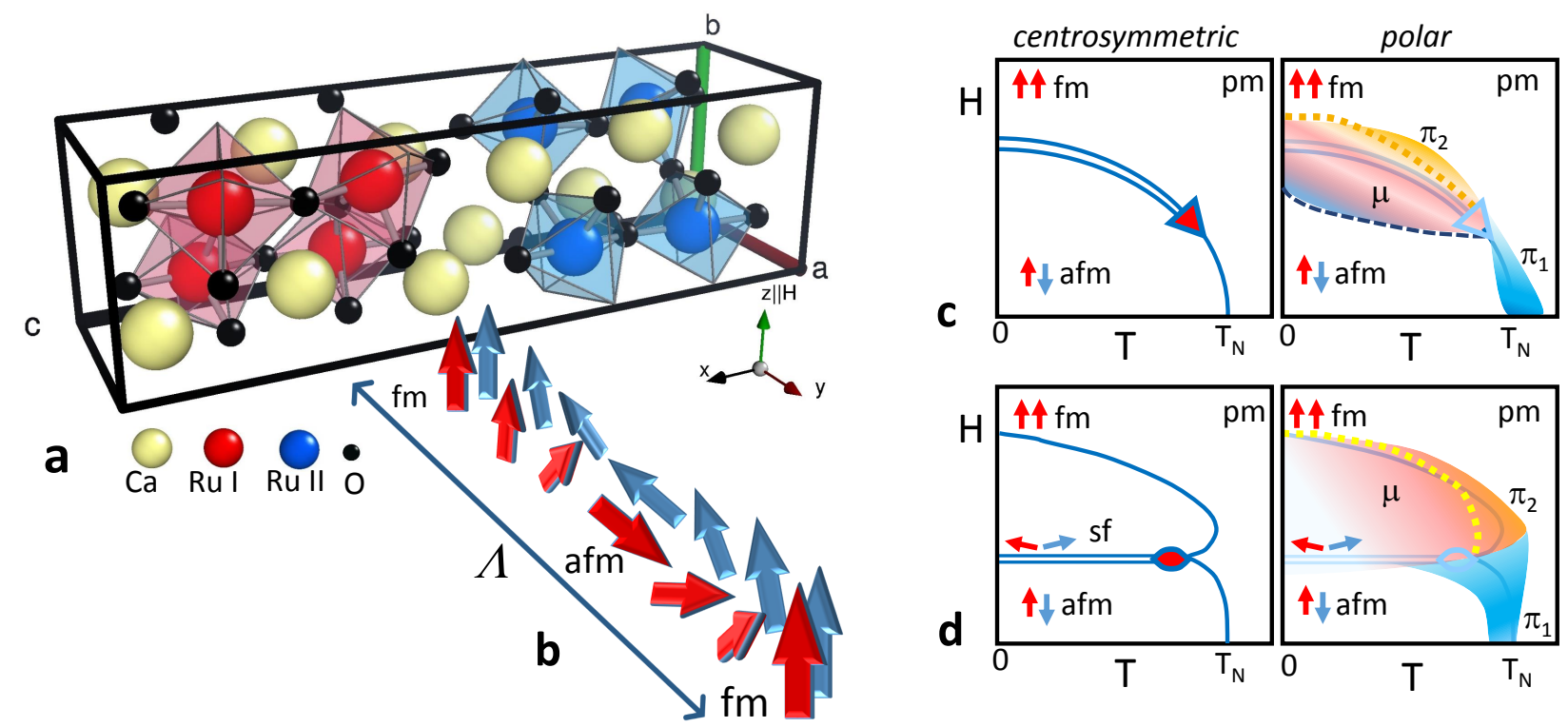

FIG. 4: Crystal structure of $\mathrm{Ca}_{3} \mathrm{Ru}_{2} \mathrm{O}_{7}$, panel a shows the The Ru atoms occupy a single crystallographic site. The antiferromagnetic primary order splits these positions into two double layers, $\mathrm{Ru}$ (I) and $\mathrm{Ru}$ (II), which have internally ferromagnetic spin-configurations and are antiparallel. Above $48 \mathrm{~K}$ transition the collinear spin-structure has moments directed along the $a$-axis of the orthorhombic cell. The polar axis is $b$. Cartesian coordinates $x y z$ are used for spin and spatial gradients, with $z$ along $b$ as indicated. Panel $\mathbf{b}$ shows a metamagnetic one-dimensional texture propagating along the $a$ axis. In an applied field along the polar axis $b$, the spin configuration oscillates from fm to afm and back to fm over a repeat period $\Lambda$. $\mathbf{c}, \mathbf{d}$, schematic phase diagrams of bipartite antiferromagnets with easy-axis anisotropy. Panel $\mathbf{c}$ the temperature-field phase diagram for the case of large anisotropy. A first-order transition between an antiferromagnetic (afm) collinear and a spin polarized (fm) state occurs along the double line. For temperatures above a tricritical point (triangle) the transition is continuous. The afm-fm co-existence can be replaced in a polar magnet by a region of modulated phases $\mu$. Towards the paramagnetic state at elevated temperatures, anomalous transitions into precursor states $\left(\pi_{1}, \pi_{2}\right)$ then are expected. The dotted yellow line indicates the transition between the improper, metamagnetic texture and the precursor state $\mu \rightarrow \pi_{2}$. The precursor of type $\pi_{1}$ only implies modulations of primary afm modes, while $\pi_{2}$ states can be metamagnetic, being composed of modulations between afm and fm modes. Panel $\mathbf{d}$ displays the phase diagram of a system with a weak anisotropy. The double line signifies a first order transition of the spin-flop type, which is only a re-orientation of the ordered moment. The marked point is a bi-critical point (almond mark). In an antiferromagnet with a polar structure, metamagnetic textures $\mu$ can still occur at elevated temperatures and for fields higher than the spin-flop field, when a sizeable net moment and antiferromagnetic order compete. As in the case of large anisotropy, different types of precursors, i.e. a proper antiferromagnetic texture $\pi_{1}$ or metamagnetic textures with coupled modulations between afm and fm mode $\pi_{2}$ can occur.

where antiferromagnetic and ferromagnetic order parameters have similar magnitude and can become intertwined. The transition from the paramagnetic state to the modulated states is expected to be unconventional, implying inhomogeneous pre-cursor states. The stabilization of this mixed magnetic texture relies on the unavoidable asymmetric exchange through spin-orbit couplings. It can be predicted for many systems to occur. So far, in $\mathrm{Ca}_{3} \mathrm{Ru}_{2} \mathrm{O}_{7}$ we have observed only a mixed state with a one-dimensional modulation. The basic mechanism that enables the generation of this mixed state can act also in different spatial directions. Then, it may become possible to create mixed textures similar to the chiral magnetic skyrmions in non-centrosymmetric ferromagnets. Therefore, appropriate non-centrosymmetric metamagnets may also bear localized or multidimensional lumps of one type order immersed in another one. Phase transition involving such textures may allow to create condensates of such lumps to form textured states that are simultaneously modulated in different directions, akin to skyrmion lattices, but composed of different co-existing ordering modes. We propose to call such states improper Dzyaloshinskii textures, as they are composed of two ordering modes with different symmetry.

\section{METHODS}

\section{A. Crystal growth and bulk characterization}

High quality single crystals of $\mathrm{Ca}_{3} \mathrm{Ru}_{2} \mathrm{O}_{7}$ were grown using a floating zone method in a mirror furnace. The single crystals were oriented using a white beam backscattering Laue $\mathrm{X}$-ray diffraction method. SFIG.5 in supplementary materials shows the corresponding Laue diffraction image indexed with the $\mathrm{Bb} 2{ }_{1} \mathrm{~m}$-structure and room temperature lattice parameters. The Laue diffraction image shows sharp reflections, which indicate the excellent quality of the sample. The crystalline quality was further confirmed by measuring the rocking curve at $(10,0,0)$ strong nuclear reflection in a neutron beam, SFIG.6. Measurements of the magnetization were performed using the vibrating sample magnetometer; specific heat was 
measured using the physical property measurements system by Quantum Design.

\section{B. X-ray diffraction and structure refinement}

As crystals of $\mathrm{Ca}_{3} \mathrm{Ru}_{2} \mathrm{O}_{7}$ are easily cleaved, great care had to be taken to isolate a single crystal of adequate quality. Finally an irregular chip $\left(162 \times 48 \times 11 \mu \mathrm{m}^{3}\right)$ was selected and used in single crystal X-ray measurements on Rigaku AFC7 diffractometer with a Saturn 724+ CCD detector. After preliminary unit cell determination oscillation images around the unit cell axes proved good crystal quality without indications of partial cleavage or twinning, see SFIG.7. All diffraction experiments were performed at $295 \mathrm{~K}$ applying graphitemonochromated Mo-K $\alpha$ radiation $(\lambda=0.71073 \AA)$ collimated with a mono-capillary. A total of three full $\varphi$-scans resulted in 2250 images from which after integration and scaling 7089 Bragg intensities were obtained. After averaging 1328 unique reflections were used in structure refinement. Derived lattice parameters, $\mathrm{a}=5.3824(6) \AA$, $\mathrm{b}=5.5254(4) \AA$, $\mathrm{c}=19.5946(15)$ $\AA$ (non-standard $B b 2_{1} m$ ) are in a very good agreement with literature data. Refinement of the established model in space group $C m c 2{ }_{1}$ (standard setting of no. 36) converged in an excellent fit of 60 parameters vs. all 1328 independent reflections. Agreement based on $\mathrm{F}^{2}$ including isotropic extinction correction is indicated by $\mathrm{wR}=0.05$ and goodness-of-fit $=$ 1.12. However, a clear assignment of the absolute structure was not possible as refinement of a twin by inversion resulted in a volume ratio of $0.45(10): 0.55$. Based on careful analyses a centrosymmetric model as well as pseudo-tetragonal twinning had to be excluded. In an ongoing investigation we try to clarify if these observations point to a structural phase transition at high temperatures and are in accordance with antiphase domain type features in optical micrographs. Details of diffraction experiment and structure determination are available from Cambridge Structural Database, CCDC 1901958.

\section{Small angle neutron scattering}

Two samples of a similar size from different growths were studied with SANS. We have carried out our SANS measurements on sample 1 using D33 SANS instrument at ILL in horizontal magnetic fields at temperatures between $10 \mathrm{~K}$ and 61 $\mathrm{K}$. The measurements were performed using unpolarized neutrons with the wavelength $\lambda=4.8 \AA$. The neutron beam was collimated over $2.8 \mathrm{~m}$ before the sample. The sample to detector distance equaled $2 \mathrm{~m}$. We have used the ILL Blue Charly 8 $\mathrm{T}$ horizontal magnet with the field oriented parallel to the neutron incident momentum. Typically, each scan was collected over 30 minutes to obtain a good statistics. The sample was cooled in a zero field to $2 \mathrm{~K}$, at which the field was applied and the data was collected on heating. The temperature was then raised to $65 \mathrm{~K}$ (above $T_{N}=56 \mathrm{~K}$ ), at which the field was reduced to zero, the sample was cooled to $2 \mathrm{~K}$ in zero field, then the next field was applied. SANS measurements on sample 2 were performed at the SANS-II instrument at PSI using a similar setup as at D33, with $\lambda=4.93 \AA$. Some of our earlier SANS measurements were performed at NG7 instrument at the NIST Center for Neutron Research.

\section{Transport and XMCD measurements}

For the transport measurements we prepared a microstructured device using a standard focused ion beam procedure. We fabricated a Hall bar device from an oriented single crystal of $\mathrm{Ca}_{3} \mathrm{Ru}_{2} \mathrm{O}_{7}$ by the application of focused ion beam (FIB) as described elsewhere[43]. We cut a thin rectangular slice, with dimensions $100 \times 20 \times 3 \mu \mathrm{m}^{3}$ from the crystal and transferred it into non-conductive epoxy on a sapphire substrate. Ohmic contacts with approximately $10 \Omega$ contact resistances were produced by sputter coating Au and annealing at $400^{\circ}$ $\mathrm{C}$. The magnetoresistance measurements were performed in a LOT Quantum design magnet system. XMCD measurements were performed on ID32 beamline at ESRF.

\section{E. Theoretical considerations}

Symmetry analysis and Landau-Ginzburg theories have been performed following standard procedures [44] with input from the ISOTROPY program (http://stokes.byu.edu/iso/isowww.php). Density-functional calculations have been performed using full-relativistic version of FPLO (https://www.fplo.de/) [45].

\section{ACKNOWLEDGEMENTS}

We thank U. Nitzsche for technical support with FPLO. N. K. acknowledges the support from JSPS KAKNHI (No. JP17H06136). D. A. S. thanks C. Geibel for the critical reading of the manuscript and constructive comments. Access to NG7 SANS was provided by the Center for High Resolution Neutron Scattering, a partnership between the National Institute of Standards and Technology and the National Science Foundation under Agreement No. DMR-1508249. We thank J. Krzywon and Y. Qiang for technical support during SANS experiment at NIST. This work is partly based on experiments performed at the Swiss spallation neutron source SINQ, Paul Scherrer Institute, Villigen, Switzerland.

\section{AUTHOR CONTRIBUTIONS}

U.K.R. conceived the project. U.K.R., A.P.M. and D.A.S. supervised the project. N.K. and D.A.S. grew single crystals. D.A.S oriented and characterised samples. H.B. and U.B. analysed the crystal structure. T.H. performed the electrical transport measurements and analysed the Hall effect data. K.K. performed XMCD measurements. D.A.S., R.C., J.S.W., and M.B. performed SANS measurements. D.A.S. and E.R. carried neutron diffraction experiments. U.K.R. carried out DFT calculations and developed the Landau-Ginzburg-type 
free energy theory. D.A.S. and U.K.R wrote the manuscript with contributions from all co-authors.

\section{DATA AVAILABILITY}

The data that support the plots within this paper may be downloaded at[46]. The datasets for the small-angle neu- tron scattering experiments on D33 are available from the Institute Laue-Langevin data portal (10.5291/ILL-DATA.5-42462)[47].
[1] Becquerel, J., van den Handel, J. Le métamagnétisme. J. Phys. Radium 10, 10-13 (1939).

[2] Becquerel, J. Le Metamagnetisme: réunion organisée en collaboration avec l'Institute de physique de l'Université de Strasbourg, Strasbourg, 21-25 Mai, 1939, Volume 1, Généralités et magnéto-optique. - Paris, p. 97-139, 1940

[3] Stryjewski, E., Giordano, N. Metamagnetism. Advances in Physics 26(5), 487-650 (1977).

[4] Néel, L. Il Nuovo Cimento 6, 942-960 (1957).

[5] Bar'yakhtar, V. G., Bogdanov, A. N. Yablonskii, D. A. The physics of magnetic domains. Usp. Fiz. Nauk 156, 47-92 (1988).

[6] Dzyaloshinskii, I. E. Theory of helicoidal structures in antiferromagnets. 1. Nonmetals. Sov. Phys. JETP 19, 960-971 (1964).

[7] Levanyuk, A. P. Incommensurate phases in dielectrics 1. (eds. R. Blinc, A. P. Levanyuk) North Holland, 1986.

[8] Cummins, H. Z. Experimental studies of structurally incommensurate crystal phases. Physics Reports 185(5-6), 211-409 (1990).

[9] De Gennes, P. G. Short range order effects in the isotropic phase of nematics and cholesterics. Molecular Crystals and Liquid Crystals 12, 193-214 (1971).

[10] Wright, D. C., Mermin, N. D. Crystalline liquids: the blue phases. Rev. Mod. Phys. 61, 385 (1989).

[11] Meiboom, S., Sethna, J. P., Anderson, P. W., Brinkman, W. F. Theory of the blue phase of cholesteric liquid crystals. Phys. Rev. Lett. 46, 1216 (1981).

[12] Nakanishi, O., Yanase, A., Hasegawa, A., Kataoka, M. The origin of the helical spin density wave in MnSi. Solid State Commun. 35(12), 995-998 (1980).

[13] Bak, P., Jensen, M. H. Theory of helical magnetic structures and phase transitions in MnSi and FeGe. J. Phys. C: Solid State Phys. 13, L881-L885 (1980).

[14] Bogdanov, A. N. New localized solutions of the nonlinear field equations. JETP Letters 62(3), 247-251 (1995).

[15] Bogdanov, A. N., Rößler, U. K., Wolf, M., Müller, K.-H. Magnetic structures and reorientation transitions in noncentrosymmetric uniaxial antiferromagnets. Phys. Rev. B 66, 214410 (2002).

[16] Bogdanov, A. N., Yablonskii, D. A. Thermodynamically stable "vortices" in magnetically ordered crystals. The mixed state of magnets. Zh. Eksp. Teor. Fiz. 95, 178-182 (1989).

[17] Rößler, U. K., Bogdanov, A. N., Pfleiderer, C. Spontaneous skyrmion ground states in magnetic metals. Nature 442, 797801 (2006).

[18] Mühlbauer, S., Binz, B., Jonietz, F., Pfleiderer, C., Rosch, A., Neubauer, A., Georgii, R. and Böni, P. Skyrmion lattice in a chiral magnet. Science 323, 915-919 (2009).

[19] Yu, X. Z., Onose, Y., Kanazawa, N., Park, J. H., Han, J. H., Matsui, Y., Nagaosa, N. and Tokura, Y. Real-space observation of a two-dimensional skyrmion crystal. Nature 465, 901-904
(2010).

[20] Jungwirth, T., Sinova, J., Manchon, A., Marti, X., Wunderlich, J., and Felser, C. The multiple directions of antiferromagnetic spintronics. Nat. Phys. 14, 200203 (2018); Gomonay, O., Baltz, V., Brataas, A., and Tserkovnyak, Y. Antiferromagnetic spin textures and dynamics. Nat. Phys. 14, 213216 (2018); Duine, R. A., Lee, Kyung-Jin, Parkin, S. S. P., and Stiles, M. D. Synthetic antiferromagnetic spintronics. Nat. Phys. 14, 217219 (2018)

[21] Yoshida, Y., Ikeda, S. I., Matsuhata, H., Shirakawa, N., Lee, C. H., Katano, S. Crystal and magnetic structure of $\mathrm{Ca}_{3} \mathrm{Ru}_{2} \mathrm{O}_{7}$. Phys. Rev. B 72, 054412 (2005).

[22] Bao, W., Mao, Z. Q., Qu, Z., Lynn, J. W. Spin Valve Effect and Magnetoresistivity in Single Crystalline $\mathrm{Ca}_{3} \mathrm{Ru}_{2} \mathrm{O}_{7}$. Phys. Rev. Lett. 100, 247203 (2008).

[23] Liu, G. Q. Mott transition and magnetic anisotropy in $\mathrm{Ca}_{3} \mathrm{Ru}_{2} \mathrm{O}_{7}$. Phys. Rev. B 84, 235137 (2011).

[24] Yoshida, Y., Nagai, I., Ikeda, S. I., Shirakawa, N., Kosaka, M., and Mori, N. Quasi-two-dimensional metallic ground state of $\mathrm{Ca}_{3} \mathrm{Ru}_{2} \mathrm{O}_{7}$. Phys. Rev. B 69, 220411(R) (2004).

[25] Nagaosa, N., Sinova, J., Onoda, S., MacDonald, A. H. and Ong, N. P. Anomalous Hall effect. Rev. Mod. Phys. 82, 1539 (2010).

[26] McCall, S., Cao, G. and Crow, J. E. Impact of magnetic fields on anisotropy in $\mathrm{Ca}_{3} \mathrm{Ru}_{2} \mathrm{O}_{7}$. Phys. Rev. B 67, 094427 (2003).

[27] Cao, G., Lin, X. N., Balicas, L., Chikara, S., Crow, J. E. and Schlottmann, P. Orbitally driven behaviour: Mott transition, quantum oscillations and colossal magnetoresistance in bilayered $\mathrm{Ca}_{3} \mathrm{Ru}_{2} \mathrm{O}_{7}$. New J. Phys. 6, 159 (2004).

[28] Fobes, D., Peng, J., Qu, Z., Liu, T. J., and Mao, Z. Q. Magnetic phase transitions and bulk spin-valve effect tuned by in-plane field orientation in $\mathrm{Ca}_{3} \mathrm{Ru}_{2} \mathrm{O}_{7}$. Phys. Rev. B 84, 014406 (2011).

[29] Dewhurst, C. D., Grillo, I., Honecker, D., Bonnaud, M., Jacques, M., Amrouni, C., Perillo-Marcone, A., Manzin, G., and Cubitt, $\mathrm{R}$. The small-angle neutron scattering instrument D33 at the Institut Laue Langevin. J. Appl. Cryst. 49, 1-14 (2015).

[30] Ke, X., Peng, J., Tian, W., Hong, T., and Mao, Z. Q. Commensurate-incommensurate magnetic phase transition in the $\mathrm{Fe}$-doped bilayer ruthenate $\mathrm{Ca}_{3} \mathrm{Ru}_{2} \mathrm{O}_{7}$. Phys. Rev. B 89, 220407(R) (2014).

[31] Zhu, M., Peng, J., Tian, W., Hong, T., Mao, Z. Q and Ke, X. Tuning the competing phases of bilayer ruthenate $\mathrm{Ca}_{3} \mathrm{Ru}_{2} \mathrm{O}_{7}$ via dilute $\mathrm{Mn}$ impurities and magnetic field. Phys. Rev. B 95, 144426 (2017).

[32] Zheludev, A., Maslov, S., Shirane, G., Sasago, S., Koide, N., Uchinokura, K. Field-induced incommensurate-tocommensurate transition in $\mathrm{Ba}_{2} \mathrm{CuGe}_{2} \mathrm{O}_{7}$. Phys. Rev. B. 57, 2968-2978 (1998).

[33] Agrestini, S., Chapon, L. C., Daoud-Aladine, A., Schefer, J., Gukasov, A., Mazzoli, C., Lees, M. R., and Petrenko, O. A., Nature of the Magnetic Order in $\mathrm{Ca}_{3} \mathrm{Co}_{2} \mathrm{O}_{6}$. Phys. Rev. Lett. 101, 097202 (2008) 
[34] Gao, S., Zaharko, O., Tsurkan, V., Su, Y., White, J. S., Tucker, G. S., Roessli, B., Bourdarot, F., Sibille, R., Chernyshov, D., Fennell, T., Loidl, A., and Rüegg, C. Spiral spin-liquid and the emergence of a vortex-like state in $\mathrm{MnSc}_{2} \mathrm{~S}_{4}$. Nat. Phys. 13, 157 (2017).

[35] Jensen, J. and Mackintosh, A. R. Rare Earth Magnetism (Clarendon Press · Oxford, 1991).

[36] Levanyuk, A. P. Thermodynamical theory of phase-transitions with appearance of an incommensurate superstructure in ferroelectrics $\mathrm{NaNO}_{2}$ and $\mathrm{SC}\left(\mathrm{NH}_{2}\right)_{2}$. Fiz. Tverd. Tela 18(7), 19271932 (1976).

[37] Stefanovskii, E. P. Exchange-relativistic modulated magneticstructures in multisublattice rhombic antiferromagnets. Fiz. Tverd. Tela 28(11), 3452-3456 (1986).

[38] Yablonskii, D. A., Medvedeva, L. I. Classification of magnetic structures in compounds with the $\mathrm{Fe}_{2} \mathrm{P}$-structure. Physica B 167(2), 125-132 (1990).

[39] Aizu, K. Investigation of Incommensurate Phases of the "Twiny" Gradient Form as Compared with Incommensurate Phases of the Simple Gradient Form. J. Phys. Soc. Jpn. 58(12), 4501-4510 (1989).

[40] Zavorotnev, Yu. D., Medvedyeva, L. I. Long-period incommensurate structures in crystals with a triangular arrangement of magnetic ions. J. Magn. Magn. Mater. 248(3), 402-412 (2002); Zavorotnev, Yu D., and Medvedeva, L. I. Character- istics of irreducible vectors rotating in superstructures with two one-component order parameters. Crystallography Reports $\mathbf{4 7}$, 1003-1006 (2002).

[41] Milward, G. C., Calderon, M. J. and Littlewood, P. B. Electronically soft phases in manganites. Nature 433, 607-611 (2005).

[42] Bar'yakhtar, V. G., Stefanovskij, E. P. and Yablonskii, D. A. Theory of magnetic structure and electric polarization of $\mathrm{Cr}_{2} \mathrm{BeO}_{4}$ system. Pisma Zh. Eksp. Teor. Fiz. 42, 258-260 (1985).

[43] Ronning, F., Helm, T, Shirer, K. R., Bachmann, M. D., Balicas, L., Chan, M. K., Ramshaw, B. J., McDonald, R. D., Balakirev, F. F., Jaime, M., Bauer, E. D., Moll, P. J. W. Electronic inplane symmetry breaking at field-tuned quantum criticality in CeRhIn $_{5}$. Nature 548, 313-317 (2017).

[44] Tolédano J. C. and Tolédano P. The Landau theory of phase transitions (World Scientific, 1987).

[45] Koepernik, K., Eschrig, H. Full-potential nonorthogonal localorbital minimum-basis band-structure scheme. Phys. Rev. B 59, 1743 (1999).

[46] https://dx.doi.org/10.17617/3.23.

[47] Sokolov D. A. and Cubitt R. (2018), Probing the magnetic field driven modulated structure and exotic magnetism in $\mathrm{Ca}_{3} \mathrm{Ru}_{2} \mathrm{O}_{7}$. (Institut Laue-Langevin, 2018) https://doi.ill.fr/10.5291/ILL-DATA.5-42-462. 


\title{
Supplementary Information for Metamagnetic texture in a polar antiferromagnet
}

\author{
D. A. Sokolov, ${ }^{1}$ N. Kikugawa, ${ }^{2}$ T. Helm,,${ }^{1}$ H. Borrmann, ${ }^{1}$ U. Burkhardt, ${ }^{1}$ R. Cubitt, ${ }^{3}$ J. S. \\ White, ${ }^{4}$ E. Ressouche, ${ }^{5}$ M. Bleuel, ${ }^{6}$ K. Kummer, ${ }^{7}$ A. P. Mackenzie, ${ }^{1,8}$ and U.K. Rößler ${ }^{9}$ \\ ${ }^{I}$ Max-Planck-Institut für Chemische Physik fester Stoffe, D-01187 Dresden, Germany \\ ${ }^{2}$ National Institute for Materials Science, Tsukuba 305-0047, Japan \\ ${ }^{3}$ Institut Laue-Langevin, 6 Rue Jules Horowitz, F-38042 Grenoble, France \\ ${ }^{4}$ Laboratory for Neutron Scattering and Imaging (LNS), \\ Paul Scherrer Institute (PSI), CH-5232 Villigen, Switzerland \\ ${ }^{5}$ Univ. Grenoble Alpes, CEA, INAC-MEM, 38000 Grenoble, France \\ ${ }^{6}$ NIST Center for Neutron Research, NIST, Gaithersburg, MD 20899, USA \\ ${ }^{7}$ ESRF, 71 avenue des Martyrs, 38000 Grenoble, France \\ ${ }^{8}$ Scottish Universities Physics Alliance (SUPA), School of Physics and Astronomy, \\ University of St Andrews, St Andrews KY16 9SS, United Kingdom \\ ${ }^{9}$ IFW Dresden, PO Box 270116, D-01171 Dresden, Germany
}

\section{X-RAY MAGNETIC CIRCULAR DICHROISM (XMCD)}

The X-ray magnetic circular dichroism (XMCD) experiment was performed at the ESRF beamline ID32 using the high-field magnet endstation ${ }^{1}$. The samples were cleaved in situ in the high field magnet at low temperature and a pressure better than $2 \times 10^{-10}$ mbar leaving behind a clean $a b$ surface. The magnetic field was applied along the beam direction. The $b$ axis of the samples was titled by $15^{\circ}$ towards the $c$ axis with respect to the field direction, resulting in a small field component along $c$ but zero field along $a$. The X-ray absorption spectra were taken in total electron yield mode with circular left and circular right polarization for both positive as well as negative field direction. The XMCD was determined from all four spectra as the difference between spectra taken with opposite helicity.

Fig. 1a shows the XAS spectra for positive and negative helicity and the XMCD obtained as the difference of the two in the CAFM phase at low temperature and high field. In addition to the $\mathrm{Ru} M_{3}$ and $M_{2}$ absorption lines there is an additional broad X-ray absorption features around $450 \mathrm{eV}$ which displays no XMCD and is due to $\mathrm{Ca} L_{3,2}$ absorption. We followed the XMCD signal at the $\mathrm{Ru} M_{3,2}$ as a function of magnetic field and temperature in the relevant region of phase space. The obtained spectra are shown in Fig. 1b both with the relative intensities as measured (left panel) and with the intensities normalized to peak value (right panel). The intensity of the XMCD signal (left panel) scales well with the macroscopic magnetization curves shown in Fig. 2a of the manuscript. The lineshape of the XMCD, however, does not change, neither with field nor temperature, across the metamagnetic, magnetic and MIT transitions. Using the XMCD sum rules, we can extract the ratio of orbital to spin moment aligned along the field which only depends on the ratio of the integrated XMCD signal at the $M_{3}$ and $M_{2}$ absorption edge, respectively ${ }^{2}$. As the XMCD lineshape does not change across the phase diagram we always find the same value $<m_{L}>/<m_{S}>\approx 0.13 \pm 0.02$, confirming the presence of a sizable orbital moment. In principle, spin- and orbital moments can also be extracted individually from the $\mathrm{XMCD}_{\text {signals }}{ }^{2}$. We refrained from doing so here because of the complex background of the XAS spectra which makes it difficult to extract reliable numbers.

The robustness of the XMCD lineshape and the $\left\langle m_{L}\right\rangle$ $/<m_{S}>$ ratio suggests that the local crystal field experienced by the $\mathrm{Ru}$ ions in the $\mathrm{RuO}_{6}$ octahedra does not change significantly in the interesting region of the phase diagram.

\section{TRANSPORT MEASUREMENTS}

We carried out magnetotransport measurements on a sample in the Hall-bar geometry, prepared by focused Ion beam (FIB) microfabrication. A lamella with dimensions $(3 \times 20 \times 100) \mu \mathrm{m}^{3}$ was cut from a single-crystal using Gallium FIB. The device is shown in the inset of Fig $2 \mathrm{a}$. We used a standard four-terminal Lock-In method for measurements of electrical resistivity. We applied a current of $100 \mu \mathrm{A}$ with frequency $\mathrm{f}=177 \mathrm{~Hz}$ along the $a$-axis while the magnetic field was applied along the $b$ axis. The zero-field resistivity curve shown in Fig 2 a resembles data reported previously ${ }^{3}$ and demonstrates the high quality of micromachined devices. The in-plane resistivity, $\rho_{x x}$, as well as the Hall resistivity, $\rho_{y x}$, exhibit a step-like behavior at low temperatures (see Fig $2 b$ and c). The sharp change evolves into a broader transition as temperature approaches $50 \mathrm{~K}$. Above $50 \mathrm{~K}$, both $\rho_{x x}$ and $\rho_{y x}$ follow an overall positive slope. Most interestingly, we observe a two-step-like behavior in $\rho_{y x}$ for temperatures between $45 \mathrm{~K}$ and $49 \mathrm{~K}$, the range in which the metamagnetic texture was observed in small-angle neutron scattering measurements. In general, the Hall resistivity can be a composition of three components:

$$
\rho_{y x}=\rho_{y x}^{N}+\rho_{y x}^{A}+\rho_{y x}^{T},
$$

where N, A, T denote the normal, anomalous and topological Hall effect contributions ${ }^{4}$. Using the expression:

$$
\frac{\rho_{y x}}{H}=R_{0}+\frac{S_{A} \rho_{x x}^{\alpha} M}{H}+\frac{\rho_{y x}^{T}}{H},
$$

where $\mathrm{H}$ is the magnetic field and $\mathrm{M}$ is the magnetization, we can extract the normal and anomalous Hall coefficients, 
(a)

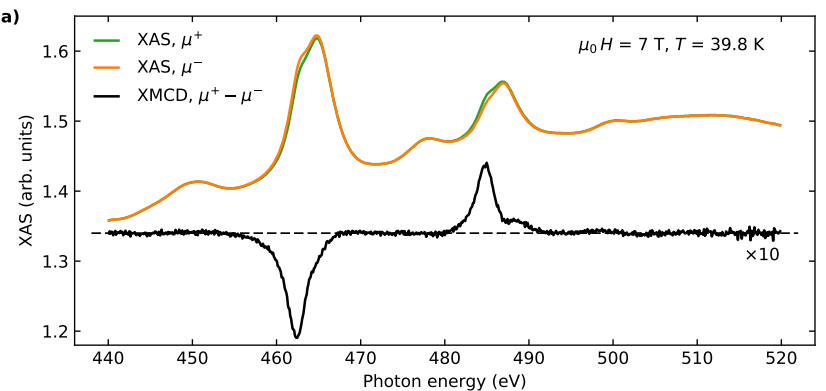

(b)
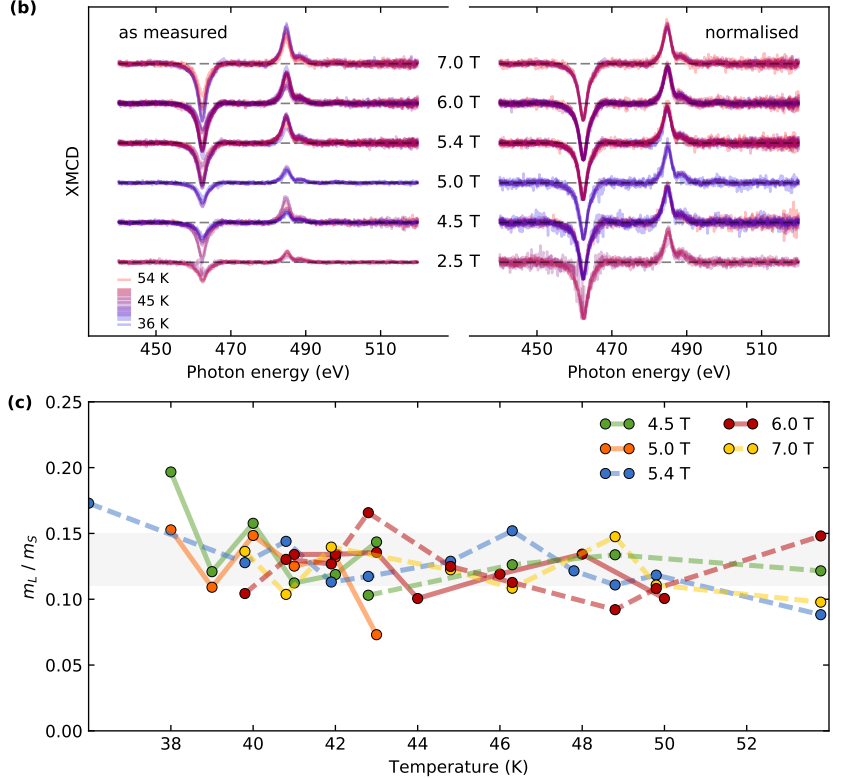

FIG. 1: (a) X-ray absorption spectra for both experimental helicities and the corresponding XMCD in the CAFM phase. (b) XMCD as a function of field and temperature in the relevant region of the phase diagram. The XMCD intensities are shown both as measured to ease comparison with macroscopic magnetization measurements (left) and normalised to average intensity to better compare the lineshapes between the XMCD spectra (right). (c) Ratio of orbital to spin moment as extracted from the XMCD data.

$\mathrm{R}_{0}$ and $\mathrm{S}_{A}$ (see Fig 3a). The respective intercept and slope of the linear fits to the high-field part of $\rho_{y x} / \mathrm{H}$ plotted versus $\mathrm{M} \rho_{x x}^{2} / \mathrm{H}$ are listed in Table 1. Again using these materials parameters and $M(H)$ data, the Hall resistivity $\rho_{y x}$ can be simulated under assumption that only the normal and anomalous contributions exist. In Fig $3 b$ we show the simulation result for $\mathrm{T}=47 \mathrm{~K}$ compared to the raw data, $\mathrm{M}, \rho_{x x}$ and $\rho_{y x}$. Both case $\alpha=0$ and 1 are shown, which correspond to an anomalous contribution dominated by intrinsic or extrinsic scattering mechanisms, respectively ${ }^{5}$. As can be seen from Fig $3 c$ deviations between simulated Hall resistivity and the experimental data appear for temperatures below $49 \mathrm{~K}$. This indicates the existence of a so-called topological contribution to $\rho_{y x}$ in the state corresponding to the $H-T$-region of the phase diagram, where the metamagnetic texture is observed. It may be related to either a topological contribution or another non-trivial contribution that is not considered through normal and anomalous Hall resistivity. In particular, it may derive from effects of a non-collinear spin-structure ${ }^{5}$.

\begin{tabular}{||ccc||}
\hline $\mathrm{T}$ & $\mathrm{R}_{0}$ & $\mathrm{~S}_{A}$ \\
\hline \hline 39 & -6.4 & $2.1 \mathrm{e}-4$ \\
\hline 41 & -7.1 & $2.5 \mathrm{e}-4$ \\
\hline 43 & -6.1 & $2.7 \mathrm{e}-4$ \\
\hline 45 & -5.1 & $2.8 \mathrm{e}-4$ \\
\hline 47 & -5.2 & $3.3 \mathrm{e}-4$ \\
\hline 49 & -4.0 & $3.2 \mathrm{e}-4$ \\
\hline 51 & 0.7 & $2.2 \mathrm{e}-4$ \\
\hline 53 & 0.63 & $2.1 \mathrm{e}-4$ \\
\hline
\end{tabular}

TABLE I: Linear fit parameters extracted from Fig 3a.

\section{SMALL-ANGLE NEUTRON SCATTERING MEASUREMENTS}

Our SANS sample 1 measured at ILL was a $238 \mathrm{mg}$ single crystal, mounted on Al sample holder with the c-axis vertical and the b-axis parallel to the field and the neutron incident momentum. The approximate sample dimensions along the major crystallographic axes were $a=8.3 \mathrm{~mm}, b=7.7 \mathrm{~mm}$, $\mathrm{c}=1.5 \mathrm{~mm}$. The SANS sample 2 measured at PSI was a 215 mg single crystal with approximate dimensions of $\mathrm{a}=6 \mathrm{~mm}$, $\mathrm{b}=5.3 \mathrm{~mm}, \mathrm{c}=2.8 \mathrm{~mm}$. The sample 2 was measured in the same orientation as sample $1 . \xi_{b}$, the correlation length of the magnetic texture along the $b$-axis, directed along the incident momentum of neutrons was calculated from the rocking curve measurement (rotation around the c-axis), schematically illustrated as $\omega$ rotation in Fig $4 \mathrm{a}$. The rocking curve at $2 \mathrm{~T}$ and $48 \mathrm{~K}$, obtained by rotating the sample together with a cryomagnet was fitted by a Gaussian lineshape, which yielded FWHM=3.1 and 2.7 degrees for the satellites contained in left and right boxes, Fig 4b,c.

$$
\xi_{b}=\frac{8 \ln 2}{Q \tan (b)}
$$

where $b$ is the full width at half maximum of the rocking curve and $\mathrm{Q}$ is the wavevector of the observed reflection. For estimates of $\xi_{a}$ and $\xi_{c}$, the correlation lengths along the $a$ and $c$ axes we used the tangential and radial widths of the satellites, which were fitted to Gaussian lineshapes. The experimental resolution in a typical SANS geometry is largely determined by the wavelength spread, $\Delta \lambda / \lambda$ and d-spacing spread of a sample, $\Delta \mathrm{d} / \mathrm{d}$. For the estimates of $\xi_{b}$ we ignored the effects of the instrumental resolution since $b>>\Delta \lambda / \lambda$ and the width of the rocking curve is much greater than the angular size of the direct beam $(\sim 0.44$ degree $)$. Here $2 \Theta$ is the scattering angle.

$$
\xi_{a}=\frac{1}{Q(\Delta d / d)}
$$

where $\Delta \mathrm{d} / \mathrm{d}$ is the $\mathrm{d}$-spacing spread of the lattice, which is 

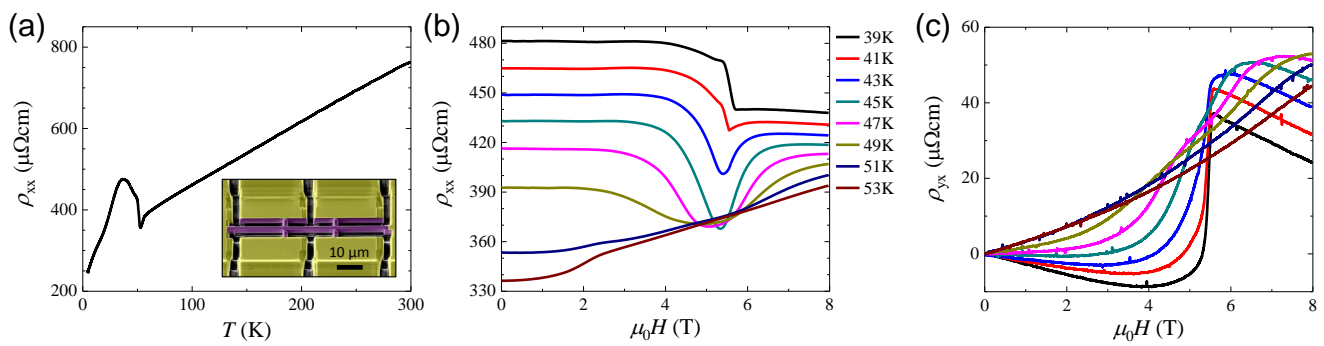

FIG. 2: Transport measurements on a microstructured single crystal of $\mathrm{Ca}_{3} \mathrm{Ru}_{2} \mathrm{O}_{7}$ : (a) Temperature dependence of the in-plane resistivity. Inset: False color SEM image of the FIB-microfabricated Hall-bar device (purple) with gold contacts (yellow). Current runs along the a-axis. (b) In-plane magnetoresistivity and (c) Hall resistivity curves at various temperatures for field applied along the b-axis.

(a)

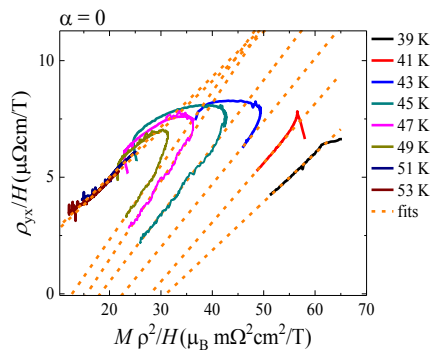

(b)

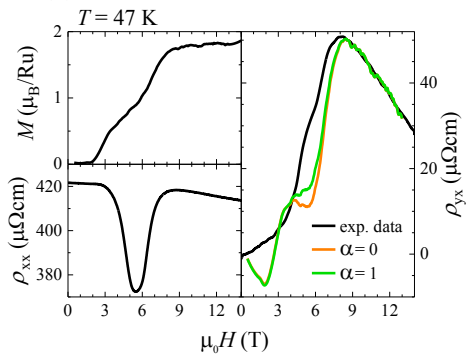

(c)

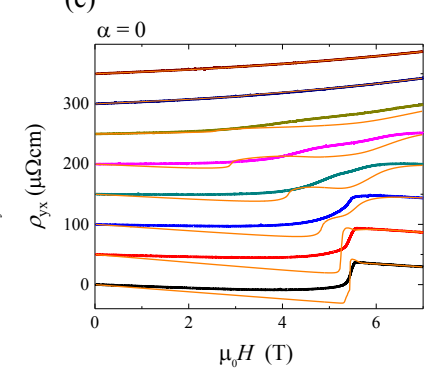

FIG. 3: (a) Linear fits (orange lines) to the Hall data from Fig 2 (c) to Eq. 2 as described in the text. The respective fit parameters we show in Table 1. (b) Example curves (black) of magnetization, in-plane resistivity and Hall resistivity, at $47 \mathrm{~K}$ used for fitting the Hall data according to Eq. 2. Pink and red curves are two simulations according to Eq. 2 with $\alpha=0,1$, respectively. (c) Hall resistivity data from Fig $2 \mathrm{c}$, offset for better visibility. Orange curves are simulations according to Eq. 2 with $\alpha=0$ using parameters from Table 1, see text for further details.

estimated from the radial width, $\mathrm{R}_{w}$ using the following,

$$
\left(R_{w} / 2 \Theta\right)^{2}=(a / 2 \Theta)^{2}+(\Delta d / d)^{2}+(\Delta \lambda / \lambda)^{2},
$$

where $a$ is the angular size of the direct beam as detailed in Ref. ${ }^{9}$.

$$
\xi_{c}=\frac{8 \ln 2}{Q \tan (t / 2)},
$$

where $t$ is the intrinsic tangential variation of the lattice, which is estimated from the tangential widths of the reflection, $T_{w}$ and direct beam, $a z$ using the following,

$$
T_{w}=\sqrt{t^{2}+a z^{2}}
$$

The field dependence of $\xi_{a}$ and $\xi_{c}$ is shown in Fig 5. Whereas no clear field dependence was observed for $\xi_{a}$, a moderate suppression of $\xi_{c}$ by the field was evidenced on both instruments. In our SANS geometry the resolution in the detector plane (our $a$-, and $c$-axes) is approximately one of order of magnitude lower than along the neutron flux direction (our $b$ axis). Therefore, the values of $\xi_{a}$ and $\xi_{c}$ should be regarded as a lower limit on correlation length in the $a c$ plane.

\section{SINGLE CRYSTAL GROWTH AND ORIENTATION OF SAMPLES}

Single crystals of $\mathrm{Ca}_{3} \mathrm{Ru}_{2} \mathrm{O}_{7}$ were grown using a floating zone method in a mirror furnace (Canon Machinery, model
SCI-MDH)), as reported elsewhere ${ }^{6}$. The crystal growth was performed in an atmosphere of the mixture of $\mathrm{Ar}$ and $\mathrm{O}_{2}$ $\left(\mathrm{Ar}: \mathrm{O}_{2}=85: 15\right)$.

The single crystals were oriented using the X-ray Laue backscattering method utilising a home-built instrument. The typical pattern shown in Fig 6 demonstrates very sharp reflections and allows to distinguish between the $a$ - and $b$-axes. The full width at the half maximum $(\mathrm{FWHM})=0.32$ degree of the rocking curve measured at the strong nuclear reflection $(100$ 0 ) in a neutron beam with the wavelength $\lambda=1.272 \AA$ on D23 instrument at ILL indicated an excellent quality of our crystal, Fig 7.

Oscillation images around crystallographic axes confirm that crystal quality was maintained for the small single crystal, Fig 8.

\section{DFT CALCULATIONS}

We have ascertained the presence of a sizeable effect of spin-orbit couplings (SOC) and orbital magnetic moments by standard DFT-calculations within the full-potential local orbital (FPLO) approach ${ }^{10}$. We used the generalized gradient approximation (GGA) as exchange-correlation functional ${ }^{11}$. Correlations beyond have been included by the GGA+U method for the $4 \mathrm{~d}$-states of $\mathrm{Ru}$ with an effective Hubbardlike $\mathrm{U}=0$ to $3 \mathrm{eV}$. The fully relativistic FPLO code includes SOC to all orders, being based on solutions of the 4- 

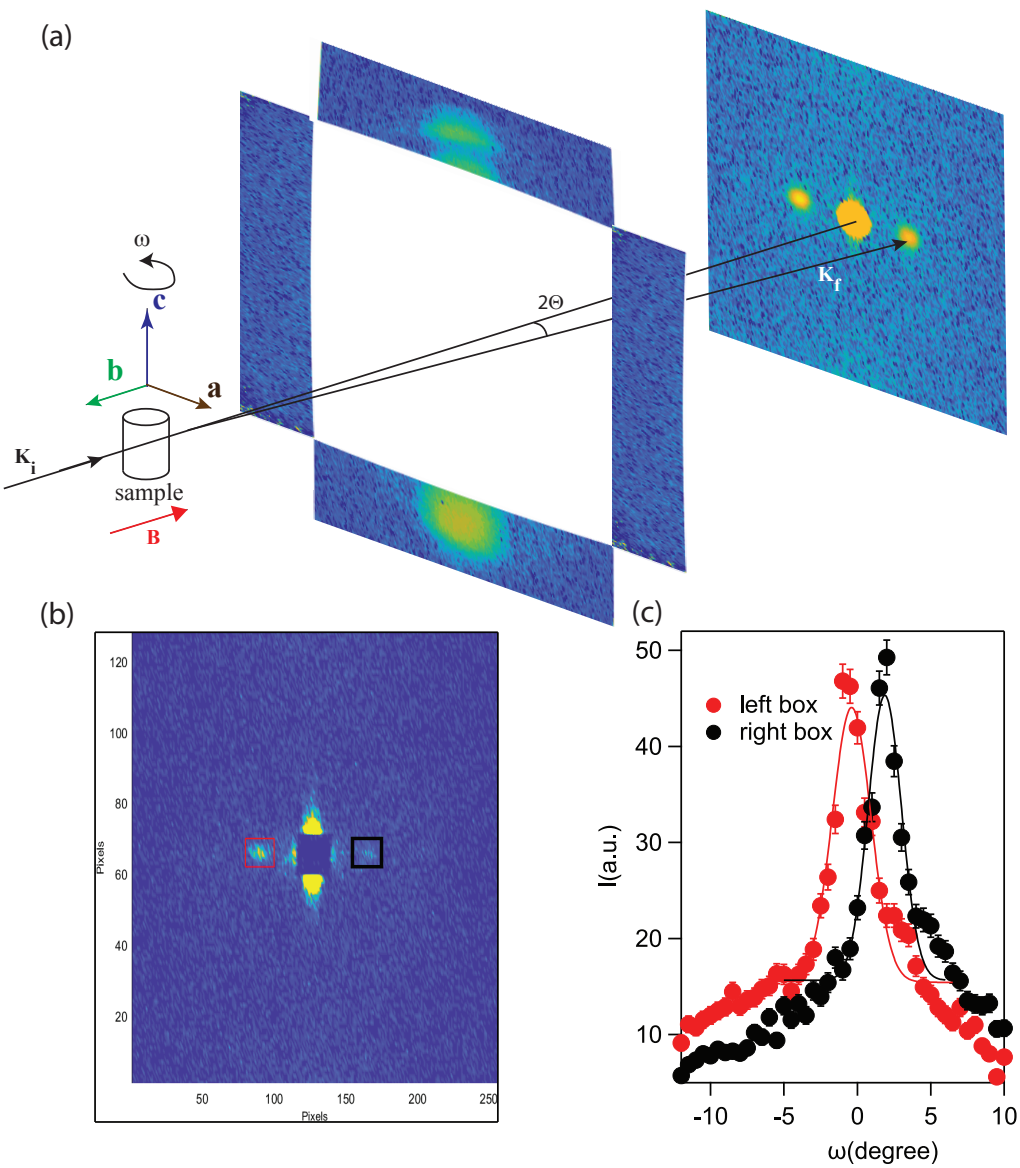

FIG. 4: (a) Geometry of SANS experiment on D33 instrument at ILL. (b) Raw SANS pattern collected at $48 \mathrm{~K}$ in magnetic field of 2 T. (c) Rocking scan of magnetic Bragg peaks shown in (b). Intensities in the left and right boxes in panel (b) are indicated by red and black markers, respectively. Solid lines are best fits to Gaussian lineshapes.
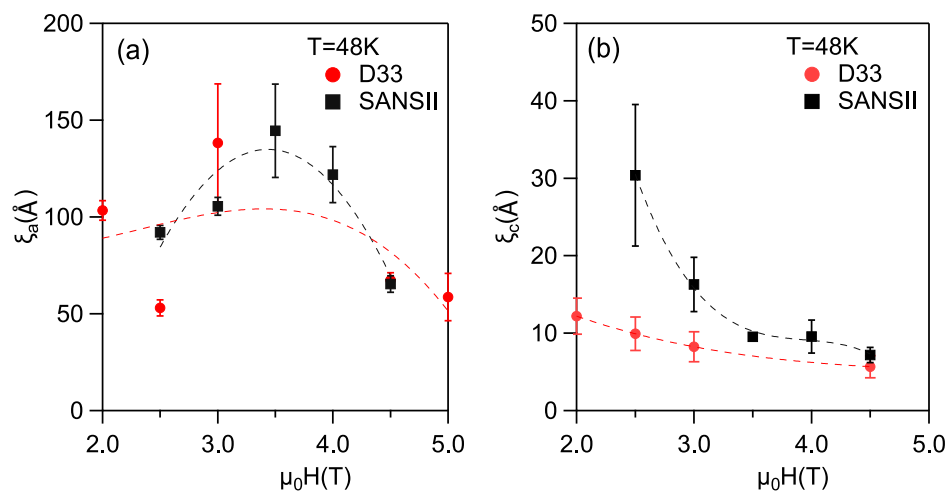

FIG. 5: Magnetic field dependence of the correlation length along the $a$ (a) and $c$-axis (b) at $48 \mathrm{~K}$. Dashed lines are a guide to the eye.

spinor Kohn-Sham-Dirac equations. As a relevant example, for ferromagnetic spin configurations we find spin-moments $m_{s}=1.56 \mu_{B} / \mathrm{Ru}$ ion and orbital moments $m_{o}=0.19 \mu_{B} /$ $\mathrm{Ru}$ ion for a representative value of $\mathrm{U}=2.25 \mathrm{eV}$. However, there are large uncertainties regarding exact values of spin and orbital moments, as seen from calculated results in Table II and the appropriate values for the DFT+U-correction in the metallic state at temperatures above the metal-insulator transition are uncertain. For $U \geq 3 \mathrm{eV}$ a gap opens and the bandstructure would correspond to an insulating ground-state, in good agreement with earlier DFT-results by Liu ${ }^{12}$. However, the results indicate the presence of relatively strong SOC effects in the collinear fully polarized state. This also suggests that antisymmetric Dzyaloshinskii-Moriya exchange is rela- 


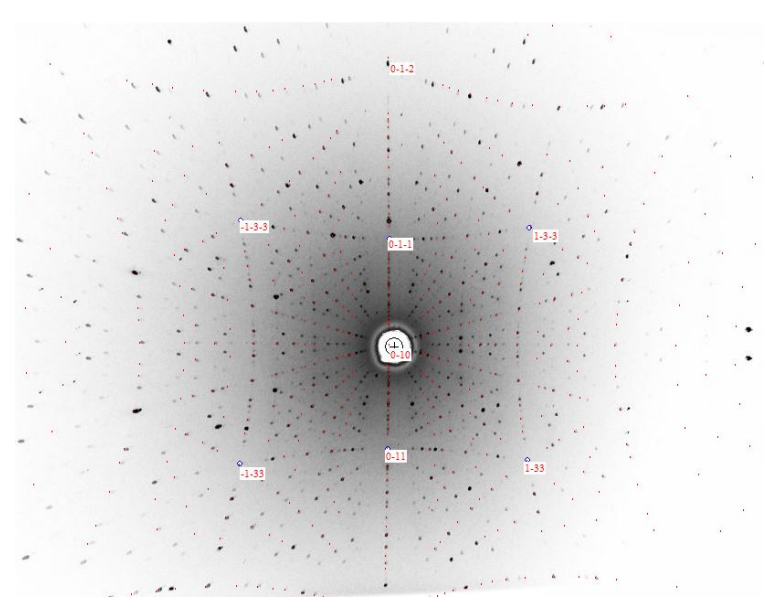

FIG. 6: The room temperature X-ray Laue diffraction pattern of an as-grown (010) facet of a $\mathrm{Ca}_{3} \mathrm{Ru}_{2} \mathrm{O}_{7}$ single crystal. The red spots and assigned Miller indices show the calculated diffraction pattern of the $\mathrm{Bb} 2_{1} \mathrm{~m}$ orthorhombic-space group.

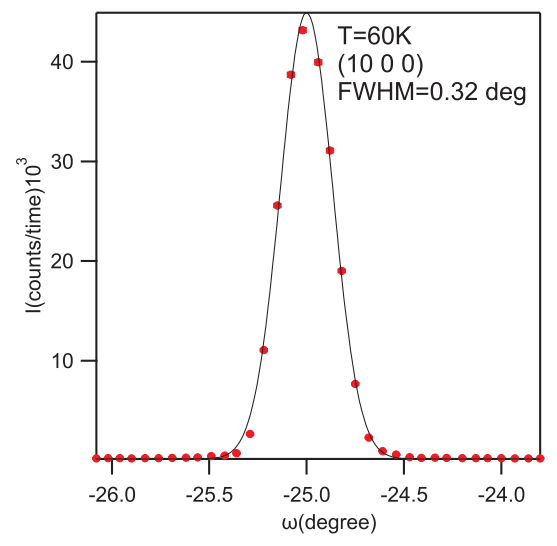

FIG. 7: The rocking curve at $(1000)$ reflection measured in a thermal neutron beam. The peak is fitted by a Gaussian lineshape with the full width at the half maximum FWHM=0.32 degree.

tively strong in $\mathrm{Ca}_{3} \mathrm{Ru}_{2} \mathrm{O}_{7}$. In view of the complex bi-layer structure of $\mathrm{Ca}_{3} \mathrm{Ru}_{2} \mathrm{O}_{7}$ and its correlated metallic character at the relevant higher temperatures, a credible microscopic evaluation of the Dzyaloshinskii-Moriya interactions (DMIs) is hardly feasible. However, as the Ru ions occupy the general 8b Wyckoff positions in $\mathrm{Ca}_{3} \mathrm{Ru}_{2} \mathrm{O}_{7}$, the microscopic DMIs between the spins $\mathbf{s}_{i}$ on these sites, $\mathbf{D}_{i j} \cdot\left(\mathbf{s}_{i} \times \mathbf{s}_{j}\right)$ are allowed for all pairs of sites with a general Dzyaloshinksii vector $\mathbf{D}_{i j}$, which is only determined by the SOC in the spin-split electronic bandstructure.

\section{LANDAU-GINZBURG FREE ENERGY}

The primary magnetic order in $\mathrm{Ca}_{3} \mathrm{Ru}_{2} \mathrm{O}_{7}$ has been identified as a simple antiferromagnetic two-sublattice structure, where ferromagnetically coupled Ru-bilayers alternate with antiparallel moments stacked in $c$-direction ${ }^{8}$. The antiferromagnetic order breaks the B-centering operation with the vector $t=(1 / 2,1 / 2,0)$ (in the Cartesian coordinate system of Fig. 4 a, which will be used for spatial and spin-coordinates

\begin{tabular}{||ccc||}
\hline $\begin{array}{c}\mathrm{U} \\
\mathrm{eV}\end{array}$ & $m_{s}$ & $\mu_{B} / \mathrm{Ru}$ \\
\hline 0 & 1.53 & 0.09 \\
1.00 & 1.46 & 0.12 \\
1.50 & 1.53 & 0.09 \\
2.00 & 1.52 & 0.20 \\
2.25 & 1.56 & 0.19 \\
3.00 & 1.74 & 0.02 \\
\hline
\end{tabular}

TABLE II: Magnetic spin moment $m_{s}$ and orbital moment $m_{o}$ on Ru in $\mathrm{Ca}_{3} \mathrm{Ru}_{2} \mathrm{O}_{7}$ from GGA+U density functional theory calculations.

in the following). The metamagnetic behavior of this twosublattice order, and the tricritical behavior Fig. 4 c, can be described by the Landau theory for the coupling of the two equivalent sublattices $M_{I}$ and $M_{I I}$, but this expansion requires higher-order terms ${ }^{13}$. In the vicinity of the tricritical point the 
(a)

(b)

(c)

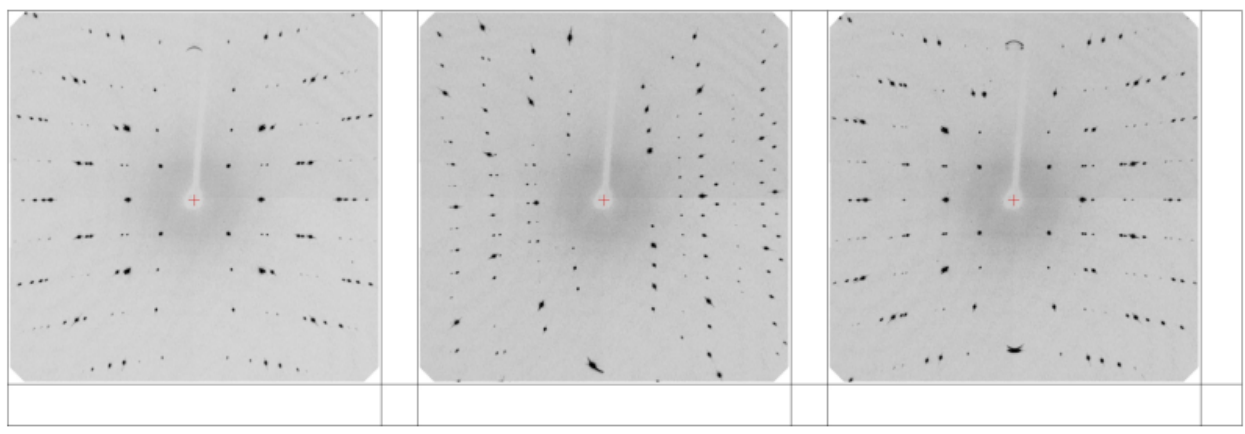

FIG. 8: (a), (b) and (c) show X-ray diffraction patterns of a small $\mathrm{Ca}_{3} \mathrm{Ru}_{2} \mathrm{O}_{7}$ crystal after rotation about the crystallographic $a, b$, and $c$-axis respectively. The rotation axis in each case is vertical.

expansion can be expressed by a free energy density

$$
\begin{aligned}
F_{0} & =B_{2}\left(\left|\mathbf{M}_{\mathrm{I}}\right|^{2}+\left|\mathbf{M}_{\mathrm{II}}\right|^{2}\right) \\
& +A_{2} \mathbf{M}_{\mathrm{I}} \cdot \mathbf{M}_{\mathrm{II}} \\
& +B_{4}\left(\left|\mathbf{M}_{\mathrm{I}}\right|^{4}+\left|\mathbf{M}_{\mathrm{II}}\right|^{4}\right) \\
& +A_{4}\left(\mathbf{M}_{\mathrm{I}} \cdot \mathbf{M}_{\mathrm{II}}\right)^{2} \\
& +B_{6}\left(\left|\mathbf{M}_{\mathrm{I}}\right|^{6}+\left|\mathbf{M}_{\mathrm{II}}\right|^{6}\right) .
\end{aligned}
$$

Representing this phenomenological theory in terms of the staggered vector $\mathbf{l}=(1 / 2)\left(\mathbf{M}_{\mathbf{I}}-\mathbf{M}_{\mathrm{II}}\right)$ of antiferromagnetism and the net magnetic moment $\mathbf{f}=(1 / 2)\left(\mathbf{M}_{\mathbf{I}}+\mathbf{M}_{\mathrm{II}}\right)$ leads to a free energy, which should include at least $6^{\text {th }}$ order terms in the Landau expansion to describe the tricritical point and the phase coexistence between antiferromagnetism and ferromagnetic field-enforced states,

$$
\begin{aligned}
w_{0} & =a_{l}|\mathbf{1}|^{2}+a_{f}|\mathbf{f}|^{2} \\
& +b_{l}|\mathbf{1}|^{4}+b_{f}|\mathbf{f}|^{4} \\
& +c_{1}|\mathbf{l}|^{2}|\mathbf{f}|^{2} \\
& +c_{l}|\mathbf{l}|^{6}+c_{f}|\mathbf{f}|^{6} \\
& +c_{2}|\mathbf{1}|^{4}|\mathbf{f}|^{2}+c_{3}|\mathbf{l}|^{2}|\mathbf{f}|^{4}
\end{aligned}
$$

The two co-existing symmetry modes $\mathbf{l}$ and $\mathbf{f}$ and their Cartesian spin-components belong to odd and even representations of the $\mathrm{Cmc} 2_{1}$ space-group (which is a standard setting of space group No36 equivalent to $\mathrm{Bb} 2{ }_{1} \mathrm{~m}$ ) with respect to the partial $t$-translation, i.e. they have different symmetry. As this crystal lattice of $\mathrm{Ca}_{3} \mathrm{Ru}_{2} \mathrm{O}_{7}$ has a non-centrosymmetric orthorhombic symmetry belonging to Laue class $2 \mathrm{~mm}$, LandauGinzburg free energies for these two modes can have Lifshitz invariants, i.e. terms linear in spatial gradients of Cartesian components of either of these modes. These terms derive from the Dzyaloshinskii-Moriya interactions and can be written as combinations of bilinear antisymmetric forms,

$$
\Gamma_{i j}^{(\gamma)}(\mathbf{x}) \equiv\left(x_{i} \partial_{\gamma} x_{j}-x_{j} \partial_{\gamma} x_{i}\right) .
$$

For the $2 \mathrm{~mm}$ symmetry and the simple antiferromagnetism in $\mathrm{Ca}_{3} \mathrm{Ru}_{2} \mathrm{O}_{7}$, the corresponding free energy contributions are

$$
\begin{aligned}
& w_{D}=D_{x} \Gamma_{z x}^{(x)}(\mathbf{l})+D_{y} \Gamma_{y y}^{(y)}(\mathbf{l}) \\
& w_{F}=F_{x} \Gamma_{z x}^{(x)}(\mathbf{f})+F_{y} \Gamma_{y z}^{(y)}(\mathbf{f}),
\end{aligned}
$$

where the coefficients $D_{x, y}, F_{x, y}$ are materials constants. These contributions, in particular $w_{D}$ lead to the spiralling cycloidal modulations of the magnetic order ${ }^{14}$, which we call Dzyaloshinskii textures. In an antiferromagnet where only the $w_{D}$ invariants are acting, an antiferromagnetic spiral would be composed only of the l-symmetry mode. Therefore, we can refer to such a sprial as a "proper" texture. In the schematic phase diagrams, Fig.4c and Fig.4d, the presence of term $w_{D}$ can lead to spiralling or other precursor textures designated $\pi_{1}$ in particular at higher temperatures above the temperature range, where the anisotropies enforce a homogeneous antiferromagnetic state, which seems to be the case in $\mathrm{Ca}_{3} \mathrm{Ru}_{2} \mathrm{O}_{7}$. Otherwise, the $w_{D}$ term can affect the antiferromagnetic order-parameter and could lead to a spiralling antiferromagnetic ground-state for weak enough anisotropies. The existence of these terms also means that the thermal phase transition from the paramagnetic to the antiferromagnetic state does not obey the Lifshitz criterion of the Landau theory for a continuous phase transition. Therefore, the thermal ordering transition in a material with a contribution of the form $w_{D}$ is expected to be anomalous. In particular, a fluctuating precursor states can arise above the magnetic ordering.

A complete phenomenological theory then requires also the usual squared gradient terms of the order-parameter,

$$
w_{E}=A_{l}(\nabla \mathbf{l})^{2}+A_{f}(\nabla \mathbf{f})^{2}+\ldots,
$$

where the ellipses stand for anisotropic exchanges terms. Indeed, in $\mathrm{Ca}_{3} \mathrm{Ru}_{2} \mathrm{O}_{7}$ strong additional anisotropies, suppress a modulation in the antiferromagnetic ground state. A complete Landau theory for the homogeneous states would require additional terms, the leading magnetocrystalline anisotropy being described by

$$
\begin{aligned}
w_{a} & =K_{z} l_{z}^{2}+k_{z} f_{z}^{2} \\
& +\kappa_{x} l_{x}^{2}+\kappa_{x y} l_{x} l_{y}+\kappa_{y} l_{y} \\
& +\nu_{x} f_{x}^{2}+\nu_{x y} l_{x} l_{y}+\nu_{y} l_{y},
\end{aligned}
$$


with anisotropy coefficients $K_{z}, \kappa, \nu$. We note that for doped compounds $\mathrm{Ca}_{3}\left(\mathrm{Ru}_{1-x} \mathrm{TM}_{x}\right)_{2} \mathrm{O}_{7}$, where TM is $\mathrm{Fe}$ or $\mathrm{Mn}$, incommensurately modulated antiferromagnetic groundstates have been observed ${ }^{15,16}$ and have been interpreted as Dzyaloshinskii spirals ${ }^{17}$. The observation suggests that the substitituion on the magnetic site weakens the anisotropy and reveals the presence of the inhomogeneous terms $w_{D}$, such that the $w_{D}$ terms overcomes the anisotropies $w_{a}$.

In the region of the metamagnetic phase co-existence, additional higher order Lifshitz terms become operative, which couple $\mathbf{l}$ and $\mathbf{f}$. There are a great number improper of couplings between these modes in $\mathrm{Ca}_{3} \mathrm{Ru}_{2} \mathrm{O}_{7}$. With the aim to illustrate the complexities of possible effects, we give here a complete list of these terms. The mixed higher order terms are Lifshitz-type invariants as follows:

$$
\left.w_{\mu}=\sum_{\alpha=x, y, z} \sum_{\beta=x, y}\left(a_{\alpha} f_{\alpha}^{2} \Gamma_{\beta z}^{(\beta)}\right)(\mathbf{l})+b_{\alpha} l_{\alpha}^{2} \Gamma_{\beta z}^{(\beta)}(\mathbf{m})\right)
$$

and

$$
\begin{aligned}
w_{\Delta} & =\Delta_{1} f_{x} f_{y} \Gamma_{x y}^{(z)}(\mathbf{l}) \\
& +\Delta_{2} f_{x} f_{y} \Gamma_{z x}^{(z)}(\mathbf{l}) \\
& +\Delta_{3} f_{x} f_{y} \Gamma_{y z}^{(x)}(\mathbf{l}) \\
& +\Delta_{4} f_{x} f_{y} \Gamma_{y z}^{(z)}(\mathbf{l}) \\
& +\Delta_{5} f_{x} f_{y} \Gamma_{z x}^{(y)}(\mathbf{l}) \\
& +\Delta_{6} f_{z} f_{x} \Gamma_{x y}^{(y)}(\mathbf{l}) \\
& +\Delta_{7} f_{z} f_{x} \Gamma_{z x}^{(z)}(\mathbf{l}) \\
& +\Delta_{8} f_{y} f_{z} \Gamma_{x y}^{(x)}(\mathbf{l}) \\
& +\Delta_{9} f_{y} f_{z} \Gamma_{y z}^{(z)}(\mathbf{l}) \\
& +\Xi_{1} l_{x} l_{y} \Gamma_{x y}^{(z)}(\mathbf{f}) \\
& +\Xi_{2} l_{x} l_{y} \Gamma_{z x}^{(z)}(\mathbf{f}) \\
& +\Xi_{3} l_{x} l_{y} \Gamma_{y z}^{(x)}(\mathbf{f}) \\
& +\Xi_{4} l_{x} l_{y} \Gamma_{y z}^{(z)}(\mathbf{f}) \\
& +\Xi_{5} l_{x} l_{y} \Gamma_{z x}^{(y)}(\mathbf{f}) \\
& +\Xi_{6} l_{z} l_{x} \Gamma_{x y}^{(y)}(\mathbf{f}) \\
& +\Xi_{7} l_{z} l_{x} \Gamma_{z x}^{(z)}(\mathbf{f}) \\
& +\Xi_{8} l_{y} l_{z} \Gamma_{x y}^{(x)}(\mathbf{f}) \\
& +\Xi_{9} l_{y} l_{z} \Gamma_{y z}^{(z)}(\mathbf{l})
\end{aligned}
$$

where coefficients $\Delta$ and $\Xi$ are materials constants. When enforced by the external field or near a multicritical point, the antiferromagnetic mode $\mathbf{l}$ and $\mathbf{f}$ can co-exist, these mixed terms become operational and will allow the formation of modulated states composed of the two different modes. Therefore, we can call these modulated states "improper textures" as they are enabled by mixed terms coupling modes of differeent symmetry.

Aditionally, there also exist higher order Lifshitz invariants that are quartic in either $\mathbf{l}, \mathbf{f}$

$$
\begin{aligned}
w_{4} & =\sum_{\alpha=x, y, z} \sum_{\beta=x, y}\left(\eta_{\alpha} f_{\alpha}^{2} \Gamma_{\beta z}^{(\beta)}(\mathbf{f})+\tau_{\alpha} l_{\alpha}^{2} \Gamma_{\beta z}^{(\beta)}(\mathbf{l})\right) \\
& +\sigma_{1} f_{x} f_{y} \Gamma_{y z}^{(x)}(\mathbf{f}) \\
& +\sigma_{2} f_{x} f_{y} \Gamma_{z x}^{(y)}(\mathbf{f}) \\
& +\sigma_{3} f_{y} f_{z} \Gamma_{z x}^{(y)}(\mathbf{f}) \\
& +\sigma_{4} f_{z} f_{x} \Gamma_{y z}^{(x)}(\mathbf{f}) \\
& +\sigma_{5} l_{x} l_{y} \Gamma_{y z}^{(x)}(\mathbf{l}) \\
& +\sigma_{6} l_{x} l_{y} \Gamma_{z x}^{(y)}(\mathbf{l}) \\
& +\sigma_{7} l_{y} l_{z} \Gamma_{z x}^{(y)}(\mathbf{l}) \\
& +\sigma_{8} l_{z} l_{x} \Gamma_{y z}^{(x)}(\mathbf{l})
\end{aligned}
$$

Depending on many materials parameters $a_{x, y z}, b_{x, y, z}, \Delta, \Xi, \eta, \tau$, and $\sigma$, these terms describe possible coupled modulations of coexisting primary symmetry modes $\mathbf{l}, \mathbf{f}$, which can take place in distinct fashion in all three spatial directions. In addition the coupling can display markedly anharmonic effects.

We observe that the Lifshitz-(type)-invariants couple different Cartesian components of the order-parameters to different spatial directions via the gradient term. Thus, these terms break isotropy in spin-space. This implies that their microscopic origin is the relativistic spin-orbit interaction. Specifically, a microscopic mechanism to explain these terms is the antisymmetric pairwise Dzyaloshinskii-Moriya exchange, or appropriate generalization for magnetic systems with a more itinerant character of spin-ordering,

A complete Landau-Ginzburg free energy density for a polar metamagnet would collect all these terms

$$
w=w_{E}+w_{0}+w_{D}+w_{F}+w_{a}+w_{\mu}+w_{\Delta}+w_{4}
$$

Minimizing this free energy functional maps out all possibilities of metamagnetic modulations around a tricritical point for the specific antiferromagnetic order parameter in space group $\mathrm{Cmc} 2{ }_{1}$. The corresponding Euler-Lagrange equations for the variational problem will constitute a system of coupled partial differential equations for the degrees of freedom described by the fields $\mathbf{l}(\mathbf{r})$, and $\mathbf{f}(\mathbf{r})$.

A dedicated theory for a specific material could be distilled from this most general functional by restricting to a few crucial terms. For a simple case, which may pertain to $\mathrm{Ca}_{3} \mathrm{Ru}_{2} \mathrm{O}_{7}$, it may suffice to consider only one-dimensional modulations in $y$-direction and spin-components in the $y z$ plane. This is the case sketched in Fig.4b. The most important terms are then proper Lifshitz invariant $\left.F_{y} \Gamma_{y z}^{(y)}\right)(\mathbf{f})$ and the mixed Lifshitz-invariants $\left.a_{z} f_{z}^{2} \Gamma_{y z}^{(y)}\right)(\mathbf{l})$ and $\left(b_{y} l_{y}^{2}+\right.$ $\left.b_{z} l_{z}^{2}\right) \Gamma_{y z}^{(y)}(\mathbf{f})$. These inhomogeneous contributions to the free energy imply that the presence of a net magnetization $f_{z}$ in an applied field along the polar axis favours an instability towards an antiferromagnetic modulation in the $y z$-plane. But, the local ferromagnetic modulation is also unstable with respect to modulations through the proper Lifshitz invariants. 
Only strong anisotropies can prevent the instability of the spin-system towards mixed states where ferromagnetic and antiferromagnetic configurations are simultaneously present in a spatially modulated fashion. The presence of these different effective couplings then leads to modulations with a competing character, as different coupling terms co-operate and frustrate each other. In our observations, this competing character of the modulation is noticable, as the characteristic modulation length displays a pronounced temperature dependence. For an ordinary Dzyaloshinskii spiral, this behavior is unusual and unexpected ${ }^{14}$, as in that case there is only one coupling term that rules the frustration of one simple symmetry mode. Also, the presence of the higher order term could lead to marked anharmonicities in metamagnetic textures that are driven by the higher order mixed terms.
For the particular antiferromagnetic order in $\mathrm{Ca}_{3} \mathrm{Ru}_{2} \mathrm{O}_{7}$, the mixed Lifshitz-type terms are of higher order and affect the magnetic spin-structure only in the region of a metamagnetic co-existence. It is the underlying tricritical point which reveals the presence of these terms, Fig.4c. However, appropriate symmetry of an antiferromagnetic mode can also allow mixed Lifshitz type invariants with a bilinear form like,

$$
C_{i j}^{(\gamma)}\left(f_{i} \partial_{\gamma} l_{j}-l_{j} \partial_{\gamma} f_{i}\right)
$$

In the vicinity of the bi-critical point, in the case of a system with weak anisotropy, sketched in Fig. 4 d, these terms drive the existence of metamagnetic textures with modulation between antiferromagnetic ground-state in spin-flopped configuration and the field-enforced ferromagnetism.
${ }^{1}$ Kummer, K. et al. The high-field magnet endstation for X-ray magnetic dichroism experiments at ESRF soft X-ray beamline ID32. J. Synchrotron Rad. 23, 464 (2016).

2 Thole, B., T. et al. X-ray circular dichroism as a probe of orbital magnetization. Phys. Rev. Lett. 68, 1943 (1992). Carra, P. et al. Xray circular dichroism and local magnetic fields. Phys. Rev. Lett. 70, 694 (1993). Chen, C., T. et al. Experimental Confirmation of the X-Ray Magnetic Circular Dichroism Sum Rules for Iron and Cobalt. Phys. Rev. Lett. 75, 152 (1995).

${ }^{3}$ Kikugawa, N., Rost, A., W., Hicks, C., W., Schofield, A., J., and Mackenzie, A., P. $\mathrm{Ca}_{3} \mathrm{Ru}_{2} \mathrm{O}_{7}$ : Density Wave Formation and Quantum Oscillations in the Hall Resistivity. J. Phys. Soc. Jpn. 79(2), 024704 (2010).

${ }^{4}$ Nagaosa, N., Sinova, J., Onoda, S., MacDonald, A. H. and Ong, N. P. Anomalous Hall effect. Rev. Mod. Phys. 82, 1539 (2010).

${ }^{5}$ Shiomi, Y., Iguchi, S., and Tokura, Y. Emergence of topological Hall effect from fanlike spin structure as modified by Dzyaloshinsky-Moriya interaction in MnP. Phys. Rev. B 86, 180404(R) (2012).

${ }^{6}$ Perry, R., S. and Maeno, Y. Systematic approach to the growth of high-quality single crystals of $\mathrm{Sr}_{3} \mathrm{Ru}_{2} \mathrm{O}_{7}$. J. Crystal Growth 271, 134 (2004)

7 Yoshida, Y., Nagai, I., Ikeda, S. I., Shirakawa, N., Kosaka, M., and Mori, N. Quasi-two-dimensional metallic ground state of $\mathrm{Ca}_{3} \mathrm{Ru}_{2} \mathrm{O}_{7}$. Phys. Rev. B 69, 220411(R) (2004).

${ }^{8}$ Yoshida Y., et al. Crystal and magnetic structure of $\mathrm{Ca}_{3} \mathrm{Ru}_{2} \mathrm{O}_{7}$.
Phys. Rev. B 72, 054412 (2005).

9 Cubitt, R., et al. Neutron diffraction by the flux lattice in high-T, superconductors. Physica B, 180 \& 181, 377 (1992).

${ }^{10}$ Koepernik, K., Eschrig, H. Full-potential nonorthogonal localorbital minimum-basis band-structure scheme. Phys. Rev. B 59, 1743 (1999).

11 Perdew, J., P., Burke, K., Ernzerhof, M. Generalized gradient approximation made simple. Phys. Rev. Lett. 77, 3865 (1996).

$12 \mathrm{Liu}, \mathrm{G}$., Q. Mott transition and magnetic anisotropy in $\mathrm{Ca}_{3} \mathrm{Ru}_{2} \mathrm{O}_{7}$. Phys. Rev. B, 84, 235137 (2011).

13 Tuszynski, J., A. Application of the Landau theory of spontaneous metamagnetism. Phys. Lett. A 85, 175 (1981).

14 Dzyaloshinskii, I. E. Sov. Phys. JETP 19, 960 (1964).

15 Ke, X., Peng, J., Tian, W., Hong, T., Zhu, M., Mao, Z. Q. Commensurate-incommensurate magnetic phase transition in the Fe-doped bilayer ruthenate $\mathrm{Ca}_{3} \mathrm{Ru}_{2} \mathrm{O}_{7}$. Phys. Rev. B, 89, 220407 (2014).

${ }^{16}$ Zhu, M., Peng, J., Tian, W., Hong, T., Mao, Z. Q and Ke, X. Tuning the competing phases of bilayer ruthenate $\mathrm{Ca}_{3} \mathrm{Ru}_{2} \mathrm{O}_{7}$ via dilute Mn impurities and magnetic field. Phys. Rev. B 95, 144426 (2017).

17 Zhu, M., Hong, T., Peng, J., Zou, T., Mao, Z. Q., Ke, X. Fieldinduced magnetic phase transitions and memory effect in bilayer ruthenate $\mathrm{Ca}_{3} \mathrm{Ru}_{2} \mathrm{O}_{7}$ with $\mathrm{Fe}$ substitution. J. Phys.: Condens. Matter 30, 075802 (2018). 